\title{
$(n, m)$-Groups in the Light of the Neutral Operations Survey Article
}

\author{
JANEZ UŠAN
}

\begin{abstract}
This text is as an attempt to systematize the results about $(n, m)-$ groups in the light of the neutral operations. (The case $m=1$ is the monograph [23].)
\end{abstract}

\section{Notion AND EXAMPles}

1.1. Definition [1]: Let $n \geq m+1(n, m \in N)$ and $(Q ; A)$ be an $(n, m)$-groupoid $\left(A: Q^{n} \rightarrow Q^{m}\right)$. We say that $(Q ; A)$ is an $(n, m)$-group iff the following statements hold:

(|) For every $i, j \in\{1, \ldots, n-m+1\}, i<j$, the following law holds

$$
A\left(x_{1}^{i-1}, A\left(x_{i}^{i+n-1}\right), x_{i+n}^{2 n-m}\right)=A\left(x_{1}^{j-1}, A\left(x_{j}^{j+n-1}\right), x_{j+n}^{2 n-m}\right)
$$

$[:<i, j>- \text { associative law }]^{1}$; and

(II) For every $i \in\{1, \ldots, n-m+1\}$ and for every $a_{1}^{n} \in Q$ there is exactly one $x_{1}^{m} \in Q^{m}$ such that the following equality holds

$$
A\left(a_{1}^{i-1}, x_{1}^{m}, a_{i}^{n-m}\right)=a_{n-m+1}^{n} .
$$

Remark: For $m=1(Q ; A)$ is an $n$-group [6]. Cf. Def. 1.1-I in [23].

1.2. Remark: A notion of an $(n, m)$-group was introduced by Ǵ. C̆upona in [1] as a generalization of the notion of a group (n-group). The paper [3] is mainly a survey on the know results for wector valued groupoids, semigroups and groups (up to 1988).

\subsection{Example [3]: Let}

2000 Mathematics Subject Classification. Primary: 20N15.

Key words and phrases. (n,m)-group, $n$-group, $\{1, n-m+1\}$-neutral operation of the $(n, m)$-groupoid.

${ }^{1}(Q ; A)$ is an $(n, m)$-semigroup. 


$$
\Phi(x, y) \stackrel{\text { def }}{=}\left(x+\frac{1}{2} \sin y, y+\frac{1}{2} \sin x\right)
$$

for all $x, y \in R$, where $R$ is the set of real numbers. Then $\Phi\left[: R^{2} \rightarrow R^{2}\right]$ is a bijection. Further on, let

$$
A(x, y, z, u) \stackrel{\text { def }}{=} \Phi^{-1}\left(x+z+\frac{1}{2}(\sin y+\sin u), y+u+\frac{1}{2}(\sin x+\sin z)\right)
$$

for all $x, y, z, u \in R$. Then $(R ; A)$ is a (4,2)-group.

\subsection{Example [3]: Let}

$$
A\left(z_{1}^{5}\right) \stackrel{\text { def }}{=}\left(z_{1}+z_{4}+\frac{1+i \sqrt{3}}{2} z_{3}, z_{2}+z_{5}+\frac{1-i \sqrt{3}}{2} z_{3}\right)
$$

for all $z_{1}^{5} \in C$, where $C$ is the set of complex numbers. Then $(C ; A)$ is a (5,2)group.

See, also [2], [3], [4] and [5].

2. $\{1, n-m+1\}-$ NEUTRAL OPERATIONS OF

$$
(n, m) \text {-GROUPOIDS }
$$

2.1. Definition [14]: Let $n \geq 2 m$ and let $(Q ; A)$ be an $(n, m)$-groupoid. Also, let $\mathbf{e}_{L}, \mathbf{e}_{R}$ and $\mathbf{e}$ be mappings of the set $Q^{n-2 m}$ into the set $Q^{m}$. Then:

1) $\mathbf{e}_{L}$ is a left $\{1, n-m+1\}$-neutral operation of the $(n, m)$-groupoid $(Q ; A)$ iff for every $x_{1}^{m} \in Q$ and for every sequence $a_{1}^{n-2 m}$ over $Q$ the following equality holds

$$
A\left(\mathbf{e}_{L}\left(a_{1}^{n-2 m}\right), a_{1}^{n-2 m}, x_{1}^{m}\right)=x_{1}^{m}
$$

2) $\mathbf{e}_{R}$ is a right $\{1, n-m+1\}$-neutral operation of the $(n, m)$-groupoid $(Q ; A)$ iff for every $x_{1}^{m} \in Q$ and for every sequence $a_{1}^{n-2 m}$ over $Q$ the following equality holds

$$
A\left(x_{1}^{m}, a_{1}^{n-2 m}, \mathbf{e}_{R}\left(a_{1}^{n-2 m}\right)\right)=x_{1}^{m} ; \text { and }
$$

3) e is a $\{1, n-m+1\}$-neutral operation of the $(n, m)$-groupoid $(Q ; A)$ iff for every $x_{1}^{m} \in Q$ and for every sequence $a_{1}^{n-2 m}$ over $Q$ the following equalities hold

$$
A\left(\mathbf{e}\left(a_{1}^{n-2 m}\right), a_{1}^{n-2 m}, x_{1}^{m}\right)=x_{1}^{m} \text { and } A\left(x_{1}^{m}, a_{1}^{n-2 m}, \mathbf{e}\left(a_{1}^{n-2 m}\right)\right)=x_{1}^{m} .
$$

Remark: For $m=1$ e is a $\{1, n\}$-neutral operation of the $n$-groupoid $(Q ; A)[13]$. For $(n, m)=(2,1), \mathbf{e}\left(a_{1}^{\circ}\right)[=\mathbf{e}(\emptyset)]$ is a neutral element of the groupoid $(Q ; A)$. Cf. Ch. II in [23].

2.2. Proposition [14]: Let $(Q ; A)$ be an $(n, m)$-groupoid and $n \geq 2 m$. Then there is at most one $\{1, n-m+1\}$-neutral operation of $(Q ; A)$. 
Proof. Suppose that $\mathbf{e}_{1}$ and $\mathbf{e}_{2}$ are $\{1, n-m+1\}$-neutral operation of an $(n, m)$-groupoid $(Q ; A)$. Then, by Def. 2.1 , for every sequence $a_{1}^{n-2 m}$ over $Q$ the following equalities hold

$$
\begin{aligned}
& A\left(\mathbf{e}_{1}\left(a_{1}^{n-2 m}\right), a_{1}^{n-2 m}, \mathbf{e}_{2}\left(a_{1}^{n-2 m}\right)\right)=\mathbf{e}_{2}\left(a_{1}^{n-2 m}\right) \text { and } \\
& A\left(\mathbf{e}_{1}\left(a_{1}^{n-2 m}\right), a_{1}^{n-2 m}, \mathbf{e}_{2}\left(a_{1}^{n-2 m}\right)\right)=\mathbf{e}_{1}\left(a_{1}^{n-2 m}\right),
\end{aligned}
$$

whence we conclude that $\mathbf{e}_{1}=\mathbf{e}_{2}$.

2.3. Proposition [14]: Let $(Q ; A)$ be an $(n, m)$-groupoid and $n \geq 2 m$. Then: if $\mathbf{e}_{L}$ is a left $\{1, n-m+1\}$-neutral operation of $(Q ; A)$ and $\mathbf{e}_{R}$ is a right $\{1, n-m+1\}$-neutral operation of $(Q ; A)$, then $\mathbf{e}_{L}=\mathbf{e}_{R}$ and $\mathbf{e}=\mathbf{e}_{L}=\mathbf{e}_{R}$ is an $\{1, n-m+1\}$-neutral operation of $(Q ; A)$.

Proof. By Def. 2.1,we conclude that for every sequence $a_{1}^{n-2 m}$ over $Q$ the following equalities hold

$$
\begin{aligned}
& A\left(\mathbf{e}_{L}\left(a_{1}^{n-2 m}\right), a_{1}^{n-2 m}, \mathbf{e}_{R}\left(a_{1}^{n-2 m}\right)\right)=\mathbf{e}_{R}\left(a_{1}^{n-2 m}\right) \text { and } \\
& A\left(\mathbf{e}_{L}\left(a_{1}^{n-2 m}\right), a_{1}^{n-2 m}, \mathbf{e}_{R}\left(a_{1}^{n-2 m}\right)\right)=\mathbf{e}_{L}\left(a_{1}^{n-2 m}\right),
\end{aligned}
$$

whence we conclude that $\mathbf{e}_{L}=\mathbf{e}_{R}$.

2.4. Proposition [19]: Let $(Q ; A)$ be an $(n, m)$-groupoid and $n \geq 2 m$. Further on, let the following statements hold:

(i) The $<1, n-m+1>-$ associative law holds in $(Q ; A)$;

(ii) For every $a_{1}^{n} \in Q$ there is at least one $x_{1}^{m} \in Q^{m}$ such that the equality $A\left(a_{1}^{n-m}, x_{1}^{m}\right)=a_{n-m+1}^{n}$ holds; and

(iii) For every $a_{1}^{n} \in Q$ there is at least one $y_{1}^{m} \in Q^{m}$ such that the equality $A\left(y_{1}^{m}, a_{1}^{n-m}\right)=a_{n-m+1}^{n}$ holds.

Then $(Q ; A)$ has a $\{1, n-m+1\}$-neutral operation.

Proof. Firstly we prove the following statements:

$1^{\circ}(Q ; A)$ has a left $\{1, n-m+1\}$-neutral operation; and

$2^{\circ}(Q ; A)$ has a right $\{1, n-m+1\}$-neutral operation.

The proof of $1^{\circ}$ :

Let $b_{1}^{m}$ be an arbitrary (fixed) sequence over $Q$. Then, by ( $i i i$ ), for every sequence $a_{1}^{n-2 m}$ over $Q$ there is at least one $\widehat{\mathbf{e}}_{L}\left(a_{1}^{n-2 m}\right) \in Q^{m}$ such that the following equality holds

$$
A\left(\widehat{\mathbf{e}}_{L}\left(a_{1}^{n-2 m}\right), a_{1}^{n-2 m}, b_{1}^{m}\right)=b_{1}^{m} .
$$

On the other hand, by $(i i)$, for every $c_{1}^{m} \in Q^{m}$ and for every sequence $k_{1}^{n-2 m}$ over $Q$ there is at least one $t_{1}^{m} \in Q^{m}$ such that the following equality holds 
(b) $\quad c_{1}^{m}=A\left(b_{1}^{m}, k_{1}^{n-2 m}, t_{1}^{m}\right)$.

By $(a),(b)$ and $(i)$, we conclude that the following series of equalities hold

$$
\begin{aligned}
A\left(\widehat{\mathbf{e}}_{L}\left(a_{1}^{n-2 m}\right), a_{1}^{n-2 m}, c_{1}^{m}\right) & \stackrel{(b)}{=} A\left(\widehat{\mathbf{e}}_{L}\left(a_{1}^{n-2 m}\right), a_{1}^{n-2 m}, A\left(b_{1}^{m}, k_{1}^{n-2 m}, t_{1}^{m}\right)\right) \\
& \stackrel{(i)}{=} A\left(A\left(\widehat{\mathbf{e}}_{L}\left(a_{1}^{n-2 m}\right), a_{1}^{n-2 m}, b_{1}^{m}\right), k_{1}^{n-2 m}, t_{1}^{m}\right) \\
& \stackrel{(a)}{=} A\left(b_{1}^{m}, k_{1}^{n-2 m}, t_{1}^{m}\right) \\
& \stackrel{(b)}{=} c_{1}^{m},
\end{aligned}
$$

whence we conclude that for every $c_{1}^{m} \in Q^{m}$ and for every sequence $a_{1}^{n-2 m}$ over $Q$ the following equality holds

$$
A\left(\widehat{\mathbf{e}}_{L}\left(a_{1}^{n-2 m}\right), a_{1}^{n-2 m}, c_{1}^{m}\right)=c_{1}^{m},
$$

i.e. that $(Q ; A)$ has the left $\{1, n-m+1\}$-neutral operation.

Similarly, it is possible to prove the statement $2^{\circ}$.

Finally, by Prop. 2.3, we conclude that there is a $\{1, n-m+1\}$-neutral operation $\mathbf{e}\left[=\widehat{\mathbf{e}}_{L}=\widehat{\mathbf{e}}_{R}\right]$.

By Prop. 2.4 and Def. 1.1, we obtain:

2.5. Theorem [14]: Every $(n, m)$-group, $n \geq 2 m$, has a $\{1, n-m+1\}$-neutral operation.

By Th. 2.5 and by Prop. 2.2, we have:

2.6. Theorem [2]: Let $(Q ; A)$ be an $(n, m)$-group and $n=2 m$. Then there is exactly one $e_{1}^{m} \in Q^{m}$ such that for all $x_{1}^{m} \in Q^{m}$ the following equalities hold

$$
A\left(x_{1}^{m}, e_{1}^{m}\right)=x_{1}^{m} \text { and } A\left(e_{1}^{m}, x_{1}^{m}\right)=x_{1}^{m} .
$$

Remark: For $m=1, e_{1}^{m}$ is a neutral element of the group $(Q ; A)$.

2.7. Theorem [2]: Let $(Q ; A)$ be a $(2 m, m)$-group and let $e_{1}^{m} \in Q^{m}$ satisfying ( $\widehat{n})$ [from Th.2.6] for all $x_{1}^{m} \in Q^{m}$. Then, for all $i \in\{0,1, \ldots, m\}$ and for every $x_{1}^{m} \in Q^{m}$ the following equality holds

$$
A\left(x_{1}^{i}, e_{1}^{m}, x_{i+1}^{m}\right)=x_{1}^{m} .
$$

Sketch of the proof. $m>1$ :

$$
\begin{aligned}
A\left(x_{1}^{i}, e_{1}^{m}, x_{i+1}^{m}\right) & \stackrel{(\widehat{n})}{=} A\left(e_{1}^{m}, A\left(x_{1}^{i}, e_{1}^{m}, x_{i+1}^{m}\right)\right) \\
& \stackrel{1.1(\mid)}{=} A\left(e_{1}^{i}, A\left(e_{i+1}^{m}, x_{1}^{i}, e_{1}^{m}\right), x_{i+1}^{m}\right) \\
& \stackrel{(\widehat{n})}{=} A\left(e_{1}^{i}, e_{i+1}^{m}, x_{1}^{i}, x_{i+1}^{m}\right) \\
& =A\left(e_{1}^{m}, x_{1}^{m}\right) \\
& \stackrel{(\widehat{n})}{=} x_{1}^{m} .
\end{aligned}
$$

By the proof of Th. 2.7, we conclude that the following proposition, also, holds: 
2.8. Theorem: Let $(Q ; A)$ be a $(2 m, m)$-semigroup and let $e_{1}^{m} \in Q^{m}$ satisfying $(\widehat{n})$ [from Th. 2.6] for all $x_{1}^{m} \in Q^{m}$. Then, for all $i \in\{0,1, \ldots, m\}$ and for every $x_{1}^{m} \in Q^{m}$ the following equality holds

$$
A\left(x_{1}^{i}, e_{1}^{m}, x_{i+1}^{m}\right)=x_{1}^{m} .
$$

2.9. Theorem [2]: Let $(Q ; A)$ be a $(2 m, m)$-group and let $e_{1}^{m} \in Q^{m}$ satisfying ( $\widehat{n})$ [from Th. 2.6] for all $x_{1}^{m} \in Q^{m}$. Then: $e_{1}=e_{2}=\cdots=e_{m}$.

Sketch of the proof. $m>1$ :

$$
\begin{aligned}
& A\left(e_{2}^{m}, e_{1}^{m}, e_{1}\right) \stackrel{2.7}{=}\left(e_{2}^{m}, e_{1}\right) \Rightarrow \\
& A\left(e_{2}^{m}, e_{1}, e_{2}^{m}, e_{1}\right)=\left(e_{2}^{m}, e_{1}\right) \stackrel{(\widehat{n})}{\Rightarrow} \\
& A\left(e_{2}^{m}, e_{1}, e_{2}^{m}, e_{1}\right)=A\left(e_{2}^{m}, e_{1}, e_{1}^{m}\right) \stackrel{1.1(\|)}{\Longrightarrow} \\
& \left(e_{2}^{m}, e_{1}\right)=\left(e_{1}^{m}\right),
\end{aligned}
$$

whence, we obtain $e_{1}=e_{2}=\cdots=e_{m}$.

2.10. Theorem [9]: Let $(Q ; A)$ be an $(n, m)$-group, e its $\{1, n-m+1\}$-neutral operation (2.1) and $n>2 m$. Then, for every $a_{1}^{n-2 m}, x_{1}^{m} \in Q$ and for all $i \in$ $\{1, \ldots, n-2 m+1\}$ the following equalities hold

$$
\begin{aligned}
& A\left(x_{1}^{m}, a_{i}^{n-2 m}, \mathbf{e}\left(a_{1}^{n-2 m}\right), a_{1}^{i-1}\right)=x_{1}^{m} \text { and } \\
& A\left(a_{i}^{n-2 m}, \mathbf{e}\left(a_{1}^{n-2 m}\right), a_{1}^{i-1}, x_{1}^{m}\right)=x_{1}^{m} .
\end{aligned}
$$

Remark: Th. 2.10 for $m=1$ is proved in [16]. Cf. Prop. 1.1-IV in [23].

Proof. Let

$$
F\left(x_{1}^{m}, b_{1}^{n-2 m}\right) \stackrel{\text { def }}{=} A\left(x_{1}^{m}, b_{i}^{n-2 m}, \mathbf{e}\left(b_{1}^{n-2 m}\right), b_{1}^{i-1}\right)
$$

for all $x_{1}^{m}, b_{1}^{n-2 m} \in Q$. Whence, we obtain

$$
\begin{aligned}
& A\left(F\left(x_{1}^{m}, b_{1}^{n-2 m}\right), b_{i}^{n-2 m}, \mathbf{e}\left(b_{1}^{n-2 m}\right), b_{1}^{i-1}\right)= \\
& A\left(A\left(x_{1}^{m}, b_{i}^{n-2 m}, \mathbf{e}\left(b_{1}^{n-2 m}\right), b_{1}^{i-1}\right), b_{i}^{n-2 m}, \mathbf{e}\left(b_{1}^{n-2 m}\right), b_{1}^{i-1}\right)
\end{aligned}
$$

for all $x_{1}^{m}, b_{1}^{n-2 m} \in Q$.

Hence, by Def. 1.1 and by Th. 2.5, we have

$$
\begin{aligned}
& A\left(F\left(x_{1}^{m}, b_{1}^{n-2 m}\right), b_{i}^{n-2 m}, \mathbf{e}\left(b_{1}^{n-2 m}\right), b_{1}^{i-1}\right)= \\
& A\left(x_{1}^{m}, b_{i}^{n-2 m}, A\left(\mathbf{e}\left(b_{1}^{n-2 m}\right), b_{1}^{i-1}, b_{i}^{n-2 m}, \mathbf{e}\left(b_{1}^{n-2 m}\right)\right), b_{1}^{i-1}\right),
\end{aligned}
$$

i.e.

$$
\begin{aligned}
& A\left(F\left(x_{1}^{m}, b_{1}^{n-2 m}\right), b_{i}^{n-2 m}, \mathbf{e}\left(b_{1}^{n-2 m}\right), b_{1}^{i-1}\right)= \\
& A\left(x_{1}^{m}, b_{i}^{n-2 m}, \mathbf{e}\left(b_{1}^{n-2 m}\right), b_{1}^{i-1}\right)
\end{aligned}
$$

for every $x_{1}^{m}, b_{1}^{n-2 m} \in Q$.

In adition, hence, by Def. 1.1 (cancelation), we obtain 


$$
F\left(x_{1}^{m}, b_{1}^{n-2 m}\right)=x_{1}^{m}
$$

for all $x_{1}^{m}, b_{1}^{n-2 m} \in Q$, whence we have (1).

Similarly, we obtain, also, (2).

2.11. Theorem [8]: Let $n>2 m, m>1,(Q ; A)$ be an $(n, m)$-group and e its $\{1, n-m+1\}$-neutral operation. Then for all $i \in\{0,1, \ldots, m\}$, for all $t \in\{1, \ldots, n-2 m+1\}$, for every $x_{1}^{m} \in Q^{m}$ and for every $a_{1}^{n-2 m} \in Q$ the following equality holds

$$
A\left(x_{1}^{i}, a_{t}^{n-2 m}, \mathbf{e}\left(a_{1}^{n-2 m}\right), a_{1}^{t-1}, x_{i+1}^{m}\right)=x_{1}^{m} .
$$

Remark: Th. 2.11 for $n=2 m$ is proved in [2]. See, also [3].

Sketch of the proof. 1) Instead of $\mathbf{e}\left(a_{1}^{n-2 m}\right)$ we are sometimes going to write

$$
\left.\overline{\mathbf{e}_{j}\left(a_{1}^{n-2 m}\right)}\right|_{j=1} ^{m} \text {. }
$$

2) $A\left(x_{1}^{i}, a_{t}^{n-2 m}, \mathbf{e}\left(a_{1}^{n-2 m}\right), a_{1}^{t-1}, x_{i+1}^{m}\right) \stackrel{(2) i=1}{=}$

$A\left(a_{1}^{n-2 m}, \mathbf{e}\left(a_{1}^{n-2 m}\right), A\left(x_{1}^{i}, a_{t}^{n-2 m}, \mathbf{e}\left(a_{1}^{n-2 m}\right), a_{1}^{t-1}, x_{i+1}^{m}\right)\right)=$

$A\left(a_{1}^{n-2 m},\left.\overline{\mathbf{e}_{j}\left(a_{1}^{n-2 m}\right)}\right|_{j=1} ^{m}, A\left(x_{1}^{i}, a_{t}^{n-2 m}, \mathbf{e}\left(a_{1}^{n-2 m}\right), a_{1}^{t-1}, x_{i+1}^{m}\right)\right)=$

$A\left(a_{1}^{n-2 m},\left.\overline{\mathbf{e}_{j}\left(a_{1}^{n-2 m}\right)}\right|_{j=1} ^{i},\left.\overline{\mathbf{e}_{j}\left(a_{1}^{n-2 m}\right)}\right|_{j=i+1} ^{m}, A\left(x_{1}^{i}, a_{t}^{n-2 m}, \mathbf{e}\left(a_{1}^{n-2 m}\right), a_{1}^{t-1}, x_{i+1}^{m}\right)\right) \stackrel{(\mid)}{=}$

$A\left(a_{1}^{n-2 m},\left.\overline{\mathbf{e}_{j}\left(a_{1}^{n-2 m}\right)}\right|_{j=1} ^{i}, A\left(\left.\overline{\mathbf{e}_{j}\left(a_{1}^{n-2 m}\right)}\right|_{j=i+1} ^{m}, x_{1}^{i}, a_{t}^{n-2 m}, \mathbf{e}\left(a_{1}^{n-2 m}\right), a_{1}^{t-1}\right), x_{i+1}^{m}\right) \stackrel{(1)}{=}$

$A\left(a_{1}^{n-2 m},\left.\overline{\mathbf{e}_{j}\left(a_{1}^{n-2 m}\right)}\right|_{j=1} ^{i},\left.\overline{\mathbf{e}_{j}\left(a_{1}^{n-2 m}\right)}\right|_{j=i+1} ^{m}, x_{1}^{i}, x_{i+1}^{m}\right)=$

$A\left(a_{1}^{n-2 m},\left.\overline{\mathbf{e}_{j}\left(a_{1}^{n-2 m}\right)}\right|_{j=1} ^{m}, x_{1}^{m}\right)$

$A\left(a_{1}^{n-2 m}, \mathbf{e}\left(a_{1}^{n-2 m}\right), x_{1}^{m}\right) \stackrel{(2) i=1}{=} x_{1}^{m}$;

$o<i<m$. [(2) and (1) from Th. 2.10.]

3. One Generalization of AN INVERSE OPERATION IN THE GRoup

3.1. Proposition [19]: Let $(Q ; A)$ be an $(n, m)$-groupoid and $n \geq 2 m$. Further on, let the statements $(i)-($ iii $)$ from Prop. 2.4 hold. Then there is mapping ${ }^{-1}$ set $Q^{n-m}$ into the set $Q^{m}$ such that the following laws

$$
\begin{aligned}
& A\left(\left(a_{1}^{n-2 m}, b_{1}^{m}\right)^{-1}, a_{1}^{n-2 m}, A\left(b_{1}^{m}, a_{1}^{n-2 m}, x_{1}^{m}\right)\right)=x_{1}^{m} \text { and } \\
& A\left(A\left(x_{1}^{m}, a_{1}^{n-2 m}, b_{1}^{m}\right), a_{1}^{n-2 m},\left(a_{1}^{n-2 m}, b_{1}^{m}\right)^{-1}\right)=x_{1}^{m}
\end{aligned}
$$

hold in the algebra $\left(Q ; A,{ }^{-1}\right)$.

Proof. Firstly we prove the following statements:

${ }^{\circ} 1$ The $<1,2 n-2 m+1>$-associative law holds in $(Q ; \stackrel{2}{A})$, where

$$
\stackrel{2}{A}\left(x_{1}^{2 n-m}\right) \stackrel{\text { def }}{=} A\left(A\left(x_{1}^{n}\right), x_{n+1}^{2 n-m}\right)
$$

for all $x_{1}^{2 n-m} \in Q$. 
${ }^{\circ} 2$ For every $a_{1}^{2 n-m} \in Q$ there is at least one $x_{1}^{m} \in Q^{m}$ such that the following equality holds

$$
\stackrel{2}{A}\left(a_{1}^{2 n-2 m}, x_{1}^{m}\right)=a_{2 n-2 m+1}^{2 n-m} .
$$

${ }^{\circ} 3$ For every $a_{1}^{2 n-m} \in Q$ there is at least one $y_{1}^{m} \in Q^{m}$ such that the following equality holds

$$
\stackrel{2}{A}\left(y_{1}^{m}, a_{1}^{2 n-2 m}\right)=a_{2 n-2 m+1}^{2 n-m} .
$$

${ }^{\circ} 4(Q ; \stackrel{2}{A})$ has a $\{1,2 n-2 m+1\}$-neutral operation.

Sketch of the proof of ${ }^{\circ} 1$ :

$$
\begin{aligned}
& \left.2 \stackrel{2}{A}\left(x_{1}^{n}, u_{1}^{n-2 m}, v_{1}^{m}\right), y_{m+1}^{n-m}, y_{n-m+1}^{n}, y_{n+1}^{2 n-m}\right)= \\
& A\left(A\left(A\left(A\left(x_{1}^{n}\right), u_{1}^{n-2 m}, v_{1}^{m}\right), y_{m+1}^{n-m}, y_{n-m+1}^{n}\right), y_{n+1}^{2 n-m}\right)= \\
& A\left(A\left(A\left(x_{1}^{n}\right), u_{1}^{n-2 m}, v_{1}^{m}\right), y_{m-1}^{n-m}, A\left(y_{n-m+1}^{2 n-m}\right)\right)= \\
& A\left(A\left(x_{1}^{n}\right), u_{1}^{n-2 m}, A\left(v_{1}^{m}, y_{m+1}^{n-m}, A\left(y_{n-m+1}^{2 n-m}\right)\right)\right)= \\
& A\left(A\left(x_{1}^{n}\right), u_{1}^{n-2 m}, A\left(A\left(v_{1}^{m}, y_{m+1}^{n-m}, y_{n-m+1}^{n}\right), y_{n+1}^{2 n-m}\right)\right)= \\
& \stackrel{2}{A}\left(x_{1}^{n}, u_{1}^{n-2 m}, \stackrel{A}{A}\left(v_{1}^{m}, y_{m+1}^{n}, y_{n+1}^{2 n-m}\right)\right) .
\end{aligned}
$$

Sketch of the proof of ${ }^{\circ} 2$ :

$\stackrel{2}{A}\left(a_{1}^{2 n-2 m}, x_{1}^{m}\right)=a_{2 n-2 m+1}^{2 n-m} \Leftrightarrow$

$A\left(A\left(a_{1}^{n}\right), a_{n+1}^{2 n-2 m}, x_{1}^{m}\right)=a_{2 n-2 m+1}^{2 n-m}$.

Sketch of the proof of ${ }^{\circ} 3$ :

$\stackrel{2}{A}\left(y_{1}^{m}, a_{1}^{2 n-2 m}\right)=a_{2 n-2 m+1}^{2 n-m} \Leftrightarrow$ $A\left(y_{1}^{m}, a_{1}^{n-2 m}, A\left(a_{n-2 m+1}^{2 n-2 m}\right)\right)=a_{2 n-2 m+1}^{2 n-m}$.

The proof of ${ }^{\circ} 4$ :

By ${ }^{\circ} 1-{ }^{\circ} 3$ and by Prop. 2.4 , we conclude that the $(2 n-m, m)-\operatorname{groupoid}(Q ; \stackrel{2}{A})$ has an $\{1,2 n-2 m+1\}$-neutral operation (let it be denoted by) $\mathrm{E}$.

In addition, let

$$
\left(a_{1}^{n-2 m}, b_{1}^{m}\right)^{-1} \stackrel{\text { def }}{=} \mathrm{E}\left(a_{1}^{n-2 m}, b_{1}^{m}, a_{1}^{n-2 m}\right)
$$

for all $a_{1}^{n-2 m}, b_{1}^{m} \in Q$. Whence, by ${ }^{\circ} 4$, we conclude that Prop. 3.1 holds.

3.2. Proposition [19]: Let $(Q ; A)$ be an $(n, m)$-groupoid and $n \geq 2 m$. Further on, let the statements $(i)-($ iii $)$ from Prop. 2.4 hold. Then there are mappings $\mathbf{e}$ and ${ }^{-1}$, respectively, of the sets $Q^{n-2 m}$ and $Q^{n-m}$ into the set $Q^{m}$ such that the following laws

$$
\begin{aligned}
& A\left(b_{1}^{m}, a_{1}^{n-2 m},\left(a_{1}^{n-2 m}, b_{1}^{m}\right)^{-1}\right)=\mathbf{e}\left(a_{1}^{n-2 m}\right) \text { and } \\
& A\left(\left(a_{1}^{n-2 m}, b_{1}^{m}\right)^{-1}, a_{1}^{n-2 m}, b_{1}^{m}\right)=\mathbf{e}\left(a_{1}^{n-2 m}\right)
\end{aligned}
$$


hold in the algebra $\left(Q ; A,{ }^{-1}, \mathbf{e}\right)$.

Proof. By Prop. 2.4 and by Prop. 3.1.

3.3. Theorem [19]: Let $(Q ; A)$ be an $(n, m)-$ group and $n \geq 2 m$. Then there are mappings e and ${ }^{-1}$, respectively, of the sets $Q^{n-2 m}$ and $Q^{n-m}$ into $Q^{m}$ such that the following laws hold in the algebra $\left(Q ; A,{ }^{-1}, \mathbf{e}\right)$

$$
\begin{aligned}
& A\left(\mathbf{e}\left(a_{1}^{n-2 m}\right), a_{1}^{n-2 m}, x_{1}^{m}\right)=x_{1}^{m}, \\
& A\left(x_{1}^{m}, a_{1}^{n-2 m}, \mathbf{e}\left(a_{1}^{n-2 m}\right)\right)=x_{1}^{m}, \\
& A\left(\left(a_{1}^{n-2 m}, b_{1}^{m}\right)^{-1}, a_{1}^{n-2 m}, b_{1}^{m}\right)=\mathbf{e}\left(a_{1}^{n-2 m}\right), \\
& A\left(b_{1}^{m}, a_{1}^{n-2 m},\left(a_{1}^{n-2 m} b_{1}^{m}\right)^{-1}\right)=\mathbf{e}\left(a_{1}^{n-2 m}\right), \\
& A\left(\left(a_{1}^{n-2 m}, b_{1}^{m}\right)^{-1}, a_{1}^{n-2 m}, A\left(b_{1}^{m}, a_{1}^{n-2 m}, x_{1}^{m}\right)\right)=x_{1}^{m} \text { and } \\
& A\left(A\left(x_{1}^{m}, a_{1}^{n-2 m}, b_{1}^{m}\right), a_{1}^{n-2 m},\left(a_{1}^{n-2 m}, b_{1}^{m}\right)^{-1}\right)=x_{1}^{m} .
\end{aligned}
$$

Proof. By Def. 1.1, Prop. 2.5, Prop. 3.1 and by Prop. 3.2.

3.4. Remark: The case $m=1$ was described in [15]. For $(n, m)=(2,1), a^{-1}$ $[=\mathrm{E}(a)]$ is the inverse element of the element a with respect to the neutral element $\mathbf{e}(\emptyset)$ of the group $(Q ; A)$. Cf. III-1 in [23].

\section{Auxiliary propositions}

4.1. Proposition [3]: Let $(Q ; A)$ be an $(n, m)$-groupoid and $n \geq m+2$. Also, let the following statements hold:

(|) $(Q ; A)$ is an $(n, m)$-semigroup;

( $(\|)$ For every $a_{1}^{n} \in Q$ there is exactly one $x_{1}^{m} \in Q^{m}$ such that the following equality holds

$$
A\left(a_{1}^{n-m}, x_{1}^{m}\right)=a_{n-m+1}^{n} ; \text { and }
$$

( $\widehat{\| I})$ For every $a_{1}^{n} \in Q$ there is exactly one $y_{1}^{m} \in Q^{m}$ such that the following equality holds

$$
A\left(y_{1}^{m}, a_{1}^{n-m}\right)=a_{n-m+1}^{n} .
$$

Then $(Q ; A)$ is an $(n, m)$-group.

\section{Sketch of the proof.}

a) $A\left(a, a_{1}^{i-1}, x_{1}^{m}, a_{i}^{n-m-2}, b\right)=A\left(a, a_{1}^{i-1}, y_{1}^{m}, a_{i}^{n-m-2}, b\right)^{2} \Rightarrow$

$$
\begin{aligned}
& A\left(c_{i+1}^{n-m}, A\left(a, a_{1}^{i-1}, x_{1}^{m}, a_{i}^{n-m-2}, b\right), c_{1}^{i}\right)= \\
& A\left(c_{i+1}^{n-m}, A\left(a, a_{1}^{i-1}, y_{1}^{m}, a_{i}^{n-m-2}, b\right), c_{1}^{i}\right) \stackrel{(())}{\Longrightarrow} \\
& A\left(A\left(c_{i+1}^{n-m}, a, a_{1}^{i-1}, x_{1}^{m}\right), a_{i}^{n-m-2}, b, c_{1}^{i}\right)=
\end{aligned}
$$

\footnotetext{
${ }^{2} i \in\{1, \ldots, n-m-1\}$.
} 


$$
\begin{aligned}
& A\left(A\left(c_{i+1}^{n-m}, a, a_{1}^{i-1}, y_{1}^{m}\right), a_{i}^{n-m-2}, b, c_{1}^{i}\right) \stackrel{(\widehat{|l|})}{\Longrightarrow} \\
& A\left(c_{i+1}^{n-m}, a, a_{1}^{i-1}, x_{1}^{m}\right)=A\left(c_{i+1}^{n-m}, a, a_{1}^{i-1}, y_{1}^{m}\right) \stackrel{(\widehat{\|})}{\Longrightarrow} \\
& x_{1}^{m}=y_{1}^{m} . \\
b) & A\left(a, a_{i}^{n-m-2}, x_{1}^{m}, a_{1}^{i-1}, b\right)=A\left(a, a_{i}^{n-m-2}, y_{1}^{m}, a_{1}^{i-1}, b\right) \Rightarrow \\
& A\left(c_{1}^{i}, A\left(a, a_{i}^{n-m-2}, x_{1}^{m}, a_{1}^{i-1}, b\right), c_{i+1}^{n-m}\right)= \\
& A\left(c_{1}^{i}, A\left(a, a_{i}^{n-m-2}, y_{1}^{m}, a_{1}^{i-1}, b\right), c_{i+1}^{n-m}\right) \stackrel{(\widehat{\mid})}{\Longrightarrow} \\
& A\left(c_{1}^{i}, a, a_{i}^{n-m-2}, A\left(x_{1}^{m}, a_{1}^{i-1}, b, c_{i+1}^{n-m}\right)\right)= \\
& A\left(c_{1}^{i}, a, a_{i}^{n-m-2}, A\left(y_{1}^{m}, a_{1}^{i-1}, b, c_{i+1}^{n-m}\right)\right) \stackrel{(\widehat{\|})}{\Longrightarrow} \\
& A\left(x_{1}^{m}, a_{1}^{i-1}, b, c_{i+1}^{n-m}\right)=A\left(y_{1}^{m}, a_{1}^{i-1}, b, c_{i+1}^{n-m}\right) \stackrel{(\widehat{\| l})}{\Longrightarrow} \\
& x_{1}^{m}=y_{1}^{m} . \\
c) & A\left(a, a_{1}^{i-1}, x_{1}^{m}, a_{i}^{n-m-2}, b\right)=b_{1}^{m} \stackrel{b)}{\Longrightarrow} \\
& A\left(c_{i+1}^{n-m}, A\left(a, a_{1}^{i-1}, x_{1}^{m}, a_{i}^{n-m-2}, b\right), c_{1}^{i}\right)=A\left(c_{i+1}^{n-m}, b_{1}^{m}, c_{1}^{i}\right) \stackrel{(\widehat{)})}{\Longleftrightarrow} \\
& A\left(A\left(c_{i+1}^{n-m}, a, a_{1}^{i-1}, x_{1}^{m}\right), a_{i}^{n-m-2}, b, c_{1}^{i}\right)=A\left(c_{i+1}^{n-m}, b_{1}^{m}, c_{1}^{i}\right),
\end{aligned}
$$

where $c_{1}^{n-m}$ is an arbitrary sequence over $Q$.

4.21. Proposition [19]: Let $n>m+1$ and let $(Q ; A)$ be an $(n, m)$-groupoid. Also, let

(a) The $<1,2>$-associative law holds in $(Q ; A)$; and

(b) For every $a_{1}^{n-m} \in Q$ and for each $x_{1}^{m}, y_{1}^{m} \in Q^{m}$ the following implication holds

$$
A\left(x_{1}^{m}, a_{1}^{n-m}\right)=A\left(y_{1}^{m}, a_{1}^{n-m}\right) \Rightarrow x_{1}^{m}=y_{1}^{m} .
$$

Then $(Q ; A)$ is an $(n, m)$-semigroup.

\section{Sketch of the proof.}

1) $i=1:(a)$.

2) $i=s$ :

$$
A\left(a_{1}^{s-1}, A\left(a_{s}^{s+n-1}\right), a_{s+n}^{2 n-m}\right)=A\left(a_{1}^{s}, A\left(a_{s+1}^{s+n}\right), a_{s+n+1}^{2 n-m}\right) .
$$

3) $s \rightarrow s+1$ :

$$
\begin{aligned}
& A\left(a_{1}^{s-1}, A\left(a_{s}^{s+n-1}\right), a_{s+n}^{2 n-m}\right)=A\left(a_{1}^{s}, A\left(a_{s+1}^{s+n}\right), a_{s+n+1}^{2 n-m}\right) \Rightarrow \\
& A\left(b_{1}, A\left(a_{1}^{s-1}, A\left(a_{s}^{s+n-1}\right), a_{s+n}^{2 n-m}\right), b_{2}^{n-m}\right)= \\
& A\left(b_{1}, A\left(a_{1}^{s}, A\left(a_{s+1}^{s+n}\right), a_{s+n+1}^{2 n-m}\right), b_{2}^{n-m}\right) \stackrel{(a)}{\Rightarrow} \\
& A\left(A\left(b_{1}, a_{1}^{s-1}, A\left(a_{s}^{s+n-1}\right), a_{s+n}^{2 n-m-1}\right), a_{2 n-m}, b_{2}^{n-m}\right)=
\end{aligned}
$$




$$
\begin{aligned}
& A\left(A\left(b_{1}, a_{1}^{s}, A\left(a_{s+1}^{s+n}\right), a_{s+n+1}^{2 n-m-1}\right), a_{2 n-m}, b_{2}^{n-m}\right) \stackrel{(b)}{\Rightarrow} \\
& A\left(b_{1}, a_{1}^{s-1}, A\left(a_{s}^{s+n-1}\right), a_{s+n}^{2 n-m-1}\right)=A\left(b_{1}, a_{1}^{s}, A\left(a_{s+1}^{s+n}\right), a_{s+n+1}^{2 n-m-1}\right) .
\end{aligned}
$$

4.2 $2_{2}$. Proposition [19]: Let $n>m+1$ and let $(Q ; A)$ be an $(n, m)$-groupoid. Also, let

$(\bar{a})$ The $<n-m, n-m+1>$-associative law holds in $(Q ; A)$; and

$(\bar{b})$ For every $a_{1}^{n-m} \in Q$ and for each $x_{1}^{m}, y_{1}^{m} \in Q^{m}$ the following implication holds

$$
A\left(a_{1}^{n-m}, x_{1}^{m}\right)=A\left(a_{1}^{n-m}, y_{1}^{m}\right) \Rightarrow x_{1}^{m}=y_{1}^{m} .
$$

Then $(Q ; A)$ is an $(n, m)$-semigroup.

The sketch of a part of the proof.

$$
\begin{aligned}
& A\left(a_{1}^{s-1}, A\left(a_{s}^{s+n-1}\right), a_{s+n}^{2 n-m}\right)=A\left(a_{1}^{s}, A\left(a_{s+1}^{s+n}\right), a_{s+n+1}^{2 n-m}\right) \Rightarrow \\
& A\left(b_{2}^{n-m}, A\left(a_{1}^{s-1}, A\left(a_{s}^{s+n-1}\right), a_{s+n}^{2 n-m}\right), b_{1}\right)= \\
& A\left(b_{2}^{n-m}, A\left(a_{1}^{s}, A\left(a_{s+1}^{s+n}\right), a_{s+n+1}^{2 n-m}\right), b_{1}\right) \stackrel{(\bar{a})}{\Rightarrow} \\
& A\left(b_{2}^{n-m}, a_{1}, A\left(a_{2}^{s-1}, A\left(a_{s}^{s+n-1}\right), a_{s+n}^{2 n-m}, b_{1}\right)\right)= \\
& A\left(b_{2}^{n-m}, a_{1}, A\left(a_{2}^{s}, A\left(a_{s+1}^{s+n}\right), a_{s+n+1}^{2 n-m}, b_{1}\right)\right) \stackrel{(\bar{b})}{\Rightarrow} \\
& A\left(a_{2}^{s-1}, A\left(a_{s}^{s+n-1}\right), a_{s+n}^{2 n-m}, b_{1}\right)=A\left(a_{2}^{s}, A\left(a_{s+1}^{s+n}\right), a_{s+n+1}^{2 n-m}, b_{1}\right) .
\end{aligned}
$$

(Cf. the proof of Prop. 4.21.

4.2. Proposition [22]: Let $n \geq m+2, i \in\{2, \ldots, n-m\}$ and let $(Q ; A)$ be an $(n, m)$-groupoid. Also, let

(i) The $<i, i+1>-$ associative law holds in $(Q ; A)$;

(ii) The $<i-1, i>-$ associative law holds in $(Q ; A)$; and

(iii) For every $a_{1}^{n-m} \in Q$ and for each $x_{1}^{m}, y_{1}^{m} \in Q^{m}$ the following implication holds

$$
A\left(a_{1}^{i-1}, x_{1}^{m}, a_{i}^{n-m}\right)=A\left(a_{1}^{i-1}, y_{1}^{m}, a_{i}^{n-m}\right) \Rightarrow x_{1}^{m}=y_{1}^{m} .
$$

Then $(Q ; A)$ is an $(n, m)$-semigroup.

The sketch of a part of of the proof. 1) Let $n=m+2(n-m=2, i=2)$.

Then, by $(i),(i i)$ and by Def. $1.1-(\mid),(Q ; A)$ is an $(n, m)$-semigroup. 
2) $i<n-m(i \in\{1, \ldots, n-m\})$ :

$$
\begin{aligned}
& A\left(a_{1}^{i-1}, A\left(a_{i}^{i+n-1}\right), a_{i+n}^{2 n-m}\right) \stackrel{(i)}{=} A\left(a_{1}^{i}, A\left(a_{i+1}^{i+n}\right), a_{i+n+1}^{2 n-m}\right) \Rightarrow \\
& A\left(c_{1}^{i}, A\left(a_{1}^{i-1}, A\left(a_{i}^{i+n-1}\right), a_{i+n}^{2 n-m}\right), c_{i+1}^{n-m}\right)= \\
& A\left(c_{1}^{i}, A\left(a_{1}^{i}, A\left(a_{i+1}^{i+n}\right), a_{i+n+1}^{2 n-m}\right), c_{i+1}^{n-m}\right) \stackrel{(i)}{\Rightarrow} \\
& A\left(c_{1}^{i-1}, A\left(c_{i}, a_{1}^{i-1}, A\left(a_{i}^{i+n-1}\right), a_{i+n}^{2 n-m-1}\right), a_{2 n-m}, c_{i+1}^{n-m}\right)= \\
& A\left(c_{1}^{i-1}, A\left(c_{i}, a_{1}^{i}, A\left(a_{i+1}^{i+n}\right), a_{i+n+1}^{2 n-m-1}\right), a_{2 n-m}, c_{i+1}^{n-m}\right) \stackrel{(i i i)}{\Rightarrow} \\
& A\left(c_{i}, a_{1}^{i-1}, A\left(a_{i}^{i+n-1}\right), a_{i+n}^{2 n-m-1}\right)=A\left(c_{i}, a_{1}^{i}, A\left(a_{i+1}^{i+n}\right), a_{i+n+1}^{2 n-m-1}\right) .
\end{aligned}
$$

3) $i>2$ :

$$
\begin{aligned}
& A\left(a_{1}^{i-2}, A\left(a_{i-1}^{i+n-2}\right), a_{i+n-1}^{2 n-m}\right) \stackrel{(i i)}{=} A\left(a_{1}^{i-1}, A\left(a_{i}^{i+n-1}\right), a_{i+n}^{2 n-m}\right) \Rightarrow \\
& A\left(c_{1}^{i-2}, A\left(a_{1}^{i-2}, A\left(a_{i-1}^{i+n-2}\right), a_{i+n-1}^{2 n-m}\right), c_{i-1}^{n-m}\right)= \\
& A\left(c_{1}^{i-2}, A\left(a_{1}^{i-1}, A\left(a_{i}^{i+n-1}\right), a_{i+n}^{2 n-m}\right), c_{i-1}^{n-m}\right) \stackrel{(i i)}{\Rightarrow} \\
& A\left(c_{1}^{i-2}, a_{1}, A\left(a_{2}^{i-2}, A\left(a_{i-1}^{i+n-2}\right), a_{i+n-1}^{2 n-m}, c_{i-1}\right), c_{i}^{n-m}\right)= \\
& A\left(c_{1}^{i-2}, a_{1}, A\left(a_{2}^{i-1}, A\left(a_{i}^{i+n-1}\right), a_{i+n}^{2 n-m}, c_{i-1}\right), c_{i}^{n-m}\right) \stackrel{(i i i)}{\Rightarrow} \\
& A\left(a_{2}^{i-2}, A\left(a_{i-1}^{i+n-2}\right), a_{i+n-1}^{2 n-m}, c_{i-1}\right)=A\left(a_{2}^{i-1}, A\left(a_{i}^{i+n-1}\right), a_{i+n}^{2 n-m}, c_{i-1}\right) .
\end{aligned}
$$

4.3. Definition: Let $(Q ; A)$ be an $(n, m)$-groupoid; $n>m$. Then:

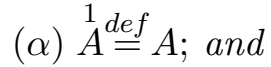
( $\beta$ ) For every $s \in N$ and for every $x_{1}^{(s+1)(n-m)+m} \in Q$$$
\stackrel{s+1}{A}\left(x_{1}^{(s+1)(n-m)+m}\right) \stackrel{\text { def }}{=} A\left(\stackrel{s}{A}\left(x_{1}^{s(n-m)+m}\right), x_{s(n-m)+m+1}^{(s+1)(n-m)+m}\right) .
$$

4.4. Proposition: Let $(Q ; A)$ be an $(n, m)$-semigroup, and $s \in N$. Then, for every $x_{1}^{(s+1)(n-m)+m} \in Q$ and for every $t \in\{1, \ldots, s(n-m)+1\}$ the following equality holds

$$
\stackrel{s+1}{A}\left(x_{1}^{(s+1)(n-m)+m}\right)=\stackrel{s}{A}\left(x_{1}^{t-1}, A\left(x_{t}^{t+n-1}\right), x_{t+n}^{(s+1)(n-m)+m}\right) .
$$

Sketch of the proof. 1) $s=1$ : By Def. $1.1-(\mid)$ and by Def. 4.3, we have

$$
\stackrel{1+1}{A}\left(x_{1}^{2(n-m)+m}\right)=\stackrel{1}{A}\left(x_{1}^{i-1}, A\left(x_{i}^{i+n-1}\right), x_{i+n}^{2(n-m)+m}\right)
$$

for every $x_{1}^{2(n-m)+m} \in Q$ and for every $i \in\{1, \ldots, n-m+1\}$.

2) $s=v$ : Let for every $x_{1}^{v(n-m)+m} \in Q$ and for all $t \in\{1, \ldots, v(n-m)+1\}$ the following equality holds

$$
\stackrel{v+1}{A}\left(x_{1}^{(v+1)(n-m)+m}\right)=\stackrel{v}{A}\left(x_{1}^{t-1}, A\left(x_{t}^{t+n-1}\right), x_{t+n}^{(v+1)(n-m)+m}\right) .
$$


3) $v \rightarrow v+1:$

$\stackrel{(v+1)+1}{A}\left(x_{1}^{(v+2)(n-m)+m}\right) \stackrel{(\beta)}{=} A\left(\stackrel{v+1}{A}\left(x_{1}^{(v+1)(n-m)+m}\right), x_{(v+1)(n-m)+m+1}^{(v+2)(n-m)+m}\right) \stackrel{2)}{=}$

$A\left(\stackrel{v}{A}\left(x_{1}^{t-1}, A\left(x_{t}^{t+n-1}\right), x_{t+n}^{(v+1)(n-m)+m}\right), x_{(v+1)(n-m)+m+1}^{(v+2)(n-m)+m}\right) \stackrel{(\beta)}{=}$

$\stackrel{v+1}{A}\left(x_{1}^{t-1}, A\left(x_{t}^{t+n-1}\right), x_{t+n}^{(v+1)(n-m)+m}, x_{(v+1)(n-m)+m+1}^{(v+2)(n-m)+m}\right) \stackrel{2)}{=}$

$\stackrel{v}{A}\left(x_{1}^{t-1}, A\left(A\left(x_{t}^{t+n-1}\right), x_{t+n}^{t+2(n-m)+m-1}, x_{t+2(n-m)+m}^{(v+2)(n-m)+m}\right) \stackrel{1.1(\mid)}{=}\right.$

$\stackrel{v}{A}\left(x_{1}^{t-1}, A\left(x_{t}^{t+i-2}, A\left(x_{t+i-1}^{t+i+n-2}\right), x_{t+i+n-1}^{t+2(n-m)+m-1}\right), x_{t+2(n-m)+m}^{(v+2)(n-m)+m}\right) \stackrel{2)}{=}$

$\left.\stackrel{v+1}{A}\left(x_{1}^{t-1}, x_{t}^{t+i-2}, A\left(x_{t+i-1}^{t+i+n-2}\right), x_{t+i+n-1}^{t+2(n-m)+m-1}\right), x_{t+2(n-m)+m}^{(v+2)(n-m)+m}\right)=$

$\stackrel{v+1}{A}\left(x_{1}^{t+i-2}, A\left(x_{t+i-1}^{t+i+n-2}\right), x_{t+i+n-1}^{(v+2)(n-m)+m}\right)$.

By Def. $1.1-(\mid)$, Def. 4.3 and Prop. 4.4, we obtain:

4.5. Proposition [1]: Let $(Q ; A)$ be an $(n, m)$-semigroup and $(i, j) \in N^{2}$. Then, for every $x_{1}^{(i+j)(n-m)+m} \in Q$ and for every $t \in\{1, \ldots, i(n-m)+1\}$ the following equality holds

$$
\stackrel{i+j}{A}\left(x_{1}^{(i+j)(n-m)+m}\right)=\stackrel{i}{A}\left(x_{1}^{t-1}, \stackrel{j}{A}\left(x_{t}^{t+j(n-m)+m-1}\right), x_{t+j(n-m)+m}^{(i+j)(n-m)+m}\right) .
$$

By Prop. 4.5 and by Def. $1.1-(\mid)$, we have:

4.6. Proposition [1]: Let $(Q ; A)$ be an $(n, m)$-semigroup and let $s \in N$. Then $(Q ; \stackrel{s}{A})$ is an $(s(n-m)+m, m)$-semigroup.

Remark: In [1] $\stackrel{s}{A}$ is written as []$_{s}$.

4.7. Proposition [1]: Let $(Q ; A)$ be an $(n, m)$-group, $n \geq 2 m$ and let $s \in N$. Then $(Q ; \stackrel{s}{A})$ is an $(s(n-m)+m, m)$-group.

Sketch of the proof. Firstly we prove the following statements:

$1^{\circ}(Q ; \stackrel{s}{A})$ is an $(s(n-m)+m, m)$-semigroup.

$2^{\circ}$ For every $a_{1}^{s(n-m)+m} \in Q$ there is exactly one $x_{1}^{m} \in Q^{m}$ such that the following equality holds

$\stackrel{s}{A}\left(a_{1}^{s(n-m)}, x_{1}^{m}\right)=a_{s(n-m)+1}^{s(n-m)+m}$.

$3^{\circ}$ For every $a_{1}^{s(n-m)+m} \in Q$ there is exactly one $y_{1}^{m} \in Q^{m}$ such that the following equality holds

$$
\stackrel{s}{A}\left(y_{1}^{m}, a_{1}^{s(n-m)}\right)=a_{s(n-m)+1}^{s(n-m)+m} .
$$


The proof of $1^{\circ}$ : By Prop. 4.6.

Sketch of the proof of $2^{\circ}$ :

$s \geq 2$ :

$\stackrel{s}{A}\left(a_{1}^{s(n-m)}, x_{1}^{m}\right)=a_{s(n-m)+1}^{s(n-m)+m} \stackrel{4.3}{\Longleftrightarrow}$
$A\left(\stackrel{s-1}{A}\left(a_{1}^{(s-1)(n-m)+m}\right), a_{(s-1)(n-m)+m+1}^{s(n-m)}, x_{1}^{m}\right)=a_{s(n-m)+1}^{s(n-m)+m}$

Sketch of the proof of $3^{\circ}$ :

$s \geq 2$ :

$\stackrel{s}{A}\left(y_{1}^{m}, a_{1}^{s(n-m)}\right)=a_{s(n-m)+1}^{s(n-m)+m} \stackrel{4.5}{\Longleftrightarrow}$

$A\left(y_{1}^{m}, a_{1}^{n-2 m}, \stackrel{s-1}{A}\left(a_{n-2 m+1}^{s(n-m)}\right)\right)=a_{s(n-m)+1}^{s(n-m)+m}$.

Finally, by $1^{\circ}-3^{\circ}$ and by Prop. 4.1, we conclude that Prop. 4.7 holds.

\section{Some Characterizations of $(n, m)$ - Groups}

5.1 . Proposition [19]: Let $n \geq 2 m, m \geq 2$ and let $(Q ; A)$ be an $(n, m)$-groupoid. Then, $(Q ; A)$ is an $(n, m)$-group iff there is a mapping ${ }^{-1}$ of the set $Q^{n-m}$ into the set $Q^{m}$ such that the laws

(1 $\left.1_{L}\right) A\left(A\left(x_{1}^{n}\right), x_{n+1}^{2 n-m}\right)=A\left(x_{1}, A\left(x_{2}^{n+1}\right), x_{n+2}^{2 n-m}\right)$,

$\left(4_{L}\right) \quad A\left(\left(a_{1}^{n-2 m}, b_{1}^{m}\right)^{-1}, a_{1}^{n-2 m}, A\left(b_{1}^{m}, a_{1}^{n-2 m}, x_{1}^{m}\right)\right)=x_{1}^{m}$ and

$\left(4_{R}\right) A\left(A\left(x_{1}^{m}, a_{1}^{n-2 m}, b_{1}^{m}\right), a_{1}^{n-2 m},\left(a_{1}^{n-2 m}, b_{1}^{m}\right)^{-1}\right)=x_{1}^{m}$

hold in the algebra $\left(Q ; A,{ }^{-1}\right)$.

Remark: For $m=1$ see IX-1 in [23].

Proof. 1) $\Rightarrow$ : By Def. 1.1 and by Th. 3.3.

$2) \Leftarrow$ : Firstly we prove the following statements:

${ }^{\circ} 1$ For every $x_{1}^{m}, y_{1}^{m} \in Q^{m}$ and for every sequence $a_{1}^{n-m}$ over $Q$ the following implication holds

$A\left(x_{1}^{m}, a_{1}^{n-m}\right)=A\left(y_{1}^{m}, a_{1}^{n-m}\right) \Rightarrow x_{1}^{m}=y_{1}^{m}$.

${ }^{\circ} 2(Q ; A)$ is an $(n, m)$-semigroup.

${ }^{\circ} 3$ For every $x_{1}^{m}, y_{1}^{m} \in Q^{m}$ and for every sequence $a_{1}^{n-m}$ over $Q$ the following implication holds

$A\left(a_{1}^{n-m}, x_{1}^{m}\right)=A\left(a_{1}^{n-m}, y_{1}^{m}\right) \Rightarrow x_{1}^{m}=y_{1}^{m}$.

${ }^{\circ} 4$ For every $x_{1}^{m}, y_{1}^{m}, b_{1}^{m}, c_{1}^{m} \in Q^{m}$ and for every sequence $a_{1}^{n-2 m}$ over $Q$ the following equivalences holds

$$
A\left(x_{1}^{m}, a_{1}^{n-2 m}, b_{1}^{m}\right)=c_{1}^{m} \Leftrightarrow x_{1}^{m}=A\left(c_{1}^{m}, a_{1}^{n-2 m},\left(a_{1}^{n-2 m}, b_{1}^{m}\right)^{-1}\right) \text { and }
$$


$A\left(b_{1}^{m}, a_{1}^{n-2 m}, y_{1}^{m}\right)=c_{1}^{m} \Leftrightarrow y_{1}^{m}=A\left(\left(a_{1}^{n-2 m}, b_{1}^{m}\right)^{-1}, a_{1}^{n-2 m}, c_{1}^{m}\right)$.

Sketch of the proof of ${ }^{\circ} 1$ :

$$
\begin{aligned}
& A\left(x_{1}^{m}, a_{1}^{n-2 m}, b_{1}^{m}\right)=A\left(y_{1}^{m}, a_{1}^{n-2 m}, b_{1}^{m}\right) \Rightarrow \\
& A\left(A\left(x_{1}^{m}, a_{1}^{n-2 m}, b_{1}^{m}\right), a_{1}^{n-2 m},\left(a_{1}^{n-2 m}, b_{1}^{m}\right)^{-1}\right)= \\
& A\left(A\left(y_{1}^{m}, a_{1}^{n-2 m}, b_{1}^{m}\right), a_{1}^{n-2 m},\left(a_{1}^{n-2 m}, b_{1}^{m}\right)^{-1}\right) \stackrel{\left(4_{R}\right)}{\longrightarrow} \\
& x_{1}^{m}=y_{1}^{m} .
\end{aligned}
$$

The proof of $2^{\circ}$ : By $(1 L),{ }^{\circ} 1$ and by Prop. $4.2_{1}$.

Sketch of the proof of ${ }^{\circ} 3$ :

$$
\begin{aligned}
& A\left(b_{1}^{m}, a_{1}^{n-2 m}, x_{1}^{m}\right)=A\left(b_{1}^{m}, a_{1}^{n-2 m}, y_{1}^{m}\right) \Rightarrow \\
& A\left(\left(a_{1}^{n-2 m}, b_{1}^{m}\right)^{-1}, a_{1}^{n-2 m}, A\left(b_{1}^{m}, a_{1}^{n-2 m}, x_{1}^{m}\right)\right)= \\
& A\left(\left(a_{1}^{n-2 m}, b_{1}^{m}\right)^{-1}, a_{1}^{n-2 m}, A\left(b_{1}^{m}, a_{1}^{n-2 m}, y_{1}^{m}\right)\right) \stackrel{\left(4_{L}\right)}{\Longrightarrow} \\
& x_{1}^{m}=y_{1}^{m} .
\end{aligned}
$$

Sketch of the proof of ${ }^{\circ} 4$ :

a) $A\left(x_{1}^{m}, a_{1}^{n-2 m}, b_{1}^{m}\right)=c_{1}^{m} \stackrel{\circ}{\Longleftrightarrow} \underset{m o n}{\Longleftrightarrow}$.

$$
\begin{aligned}
& A\left(A\left(x_{1}^{m}, a_{1}^{n-2 m}, b_{1}^{m}\right), a_{1}^{n-2 m},\left(a_{1}^{n-2 m}, b_{1}^{m}\right)^{-1}\right)= \\
& A\left(c_{1}^{m}, a_{1}^{n-2 m},\left(a_{1}^{n-2 m}, b_{1}^{m}\right)^{-1}\right) \stackrel{\left(4_{R}\right)}{\Longleftrightarrow} \\
& x_{1}^{m}=A\left(c_{1}^{m}, a_{1}^{n-2 m},\left(a_{1}^{n-2 m}, b_{1}^{m}\right)^{-1}\right) .
\end{aligned}
$$

b) $A\left(b_{1}^{m}, a_{1}^{n-2 m}, y_{1}^{m}\right)=c_{1}^{m} \stackrel{\circ}{\Longleftrightarrow}$

$$
\begin{aligned}
& A\left(\left(a_{1}^{n-2 m}, b_{1}^{m}\right)^{-1}, a_{1}^{n-2 m}, A\left(b_{1}^{m}, a_{1}^{n-2 m}, y_{1}^{m}\right)\right)= \\
& \left.A\left(\left(a_{1}^{n-2 m}, b_{1}^{m}\right)^{-1}, a_{1}^{n-2 m}, c_{1}^{m}\right)\right) \stackrel{\left(4_{L}\right)}{\Longleftrightarrow} \\
& y_{1}^{m}=A\left(\left(a_{1}^{n-2 m}, b_{1}^{m}\right)^{-1}, a_{1}^{n-2 m}, c_{1}^{m}\right) .
\end{aligned}
$$

Finally, by ${ }^{\circ} 1-{ }^{\circ} 4$ and by Prop. 4.1 , we conclude that $(Q ; A)$ is an $(n, m)$-group. Whence, by " $\Rightarrow$ ", we obtain Th. 5.1 .

Similarly, it is posible to prove also the following proposition:

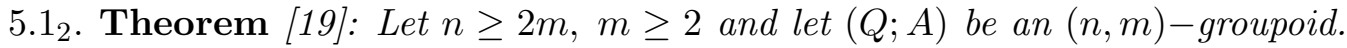
Then, $(Q ; A)$ is an $(n, m)$-group iff there is a mapping ${ }^{-1}$ of the set $Q^{n-m}$ into the set $Q^{m}$ such that the laws

$\left(1_{R}\right) A\left(x_{1}^{n-m-1}, A\left(x_{n-m}^{2 n-m-1}\right), x_{2 n-m}\right)=A\left(x_{1}^{n-m}, A\left(x_{n-m+1}^{2 n-m}\right)\right)$,

$\left(4_{L}\right) \quad A\left(\left(a_{1}^{n-2 m}, b_{1}^{m}\right)^{-1}, a_{1}^{n-2 m}, A\left(b_{1}^{m}, a_{1}^{n-2 m}, x_{1}^{m}\right)\right)=x_{1}^{m}$ and

$\left(4_{R}\right) A\left(A\left(x_{1}^{m}, a_{1}^{n-2 m}, b_{1}^{m}\right), a_{1}^{n-2 m},\left(a_{1}^{n-2 m}, b_{1}^{m}\right)^{-1}\right)=x_{1}^{m}$

hold in the algebra $\left(Q ; A,{ }^{-1}\right)$. 
Remark: For $m=1$ see IX-1 in [23].

5.21. Theorem [21]: Let $(Q ; A)$ be an $(n, m)$-groupoid, $m \geq 2$ and $n \geq 2 m$. Then: $(Q ; A)$ is an $(n, m)$-group iff the following statements hold:

(1) The $<1,2>-$ associative law holds in $(Q ; A)$;

(2) The $<1, n-m+1>-$ associative law holds in $(Q ; A)$;

(3) For every $a_{1}^{n} \in Q$ there is at least one $x_{1}^{m} \in Q^{m}$ such that the following equality

$$
A\left(a_{1}^{n-m}, x_{1}^{m}\right)=a_{n-m+1}^{n}
$$

holds; and

(4) For every $a_{1}^{n} \in Q$ there $i s$ at least one $y_{1}^{m} \in Q^{m}$ such that the following equality

$$
A\left(y_{1}^{m}, a_{1}^{n-m}\right)=a_{n-m+1}^{n}
$$

holds.

Remark: For $m=1$ Prop. $5.1_{1}$ is proved in [18]. See, also Chapter IX in [Ušan 2003]; 3.1-3.3.

Proof. $a) \Rightarrow$ : By Def. 1.1.

b) $\Leftarrow$ : Firstly we prove the following statement:

$1^{\circ}$ There is mapping ${ }^{-1}$ of the set $Q^{n-m}$ into the set $Q^{m}$ such that the following laws hold in the algebra $\left(Q ; A,{ }^{-1}\right)$ [of the type $<n, n-1>$ ]

(a) $A\left(\left(a_{1}^{n-2 m}, b_{1}^{m}\right)^{-1}, a_{1}^{n-2 m}, A\left(b_{1}^{m}, a_{1}^{n-2 m}, x_{1}^{m}\right)\right)=x_{1}^{m}$ and

(b) $A\left(A\left(x_{1}^{m}, a_{1}^{n-2 m}, b_{1}^{m}\right), a_{1}^{n-2 m},\left(a_{1}^{n-2 m}, b_{1}^{m}\right)^{-1}\right)=x_{1}^{m}$.

The proof of $1^{\circ}$ : By (2)-(4) and by Prop. 3.1.

Finally, by $(1)$, by $1^{\circ}$ and by Th. $5.1_{1}$, we conclude that $(Q ; A)$ is an $(n, m)$-group. Whence, by " $\Rightarrow "$, we obtain Th. $5.2_{1}$.

Similarly, it is posible to prove also the following proposition:

5.2. . Theorem [21]: Let $(Q ; A)$ be an $(n, m)$-groupoid, $m \geq 2$ and $n \geq 2 m$.

Then: $(Q ; A)$ is an $(n, m)$-group iff the following statements hold:

(1) The $<n-m, n-m+1>-$ associative law holds in $(Q ; A)$;

$(\overline{2})$ The $<1, n-m+1>-$ associative law holds in $(Q ; A)$;

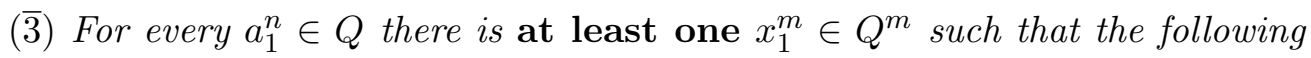
equality

$$
A\left(a_{1}^{n-m}, x_{1}^{m}\right)=a_{n-m+1}^{n}
$$

holds; and 
(4) For every $a_{1}^{n} \in Q$ there is at least one $y_{1}^{m} \in Q^{m}$ such that the following equality

$$
A\left(y_{1}^{m}, a_{1}^{n-m}\right)=a_{n-m+1}^{n}
$$

holds.

5.31. Theorem [19]: Let $n \geq 2 m, m \geq 2$ and let $(Q ; A)$ be an $(n, m)$-groupoid. Then, $(Q ; A)$ is an $(n, m)$-group iff there are mappings ${ }^{-1}$ and $\mathbf{e}$, respectively, of the sets $Q^{n-m}$ and $Q^{n-2 m}$ into the set $Q^{m}$ such that the laws

(1 $\left.1_{L}\right) A\left(A\left(x_{1}^{n}\right), x_{n+1}^{2 n-m}\right)=A\left(x_{1}, A\left(x_{2}^{n+1}\right), x_{n+2}^{2 n-m}\right)$,

$\left(2_{L}\right) \quad A\left(\mathbf{e}\left(a_{1}^{n-2 m}\right), a_{1}^{n-2 m}, x_{1}^{m}\right)=x_{1}^{m}$ and

$\left(4_{R}\right) \quad A\left(A\left(x_{1}^{m}, a_{1}^{n-2 m}, b_{1}^{m}\right), a_{1}^{n-2 m},\left(a_{1}^{n-2 m}, b_{1}^{m}\right)^{-1}\right)=x_{1}^{m}$

hold in the algebra $\left(Q ; A,{ }^{-1}, \mathbf{e}\right)$.

Remark: For $m=1$ Th. $5.3_{1}$ is proved in [17]. Cf. Chapter III in [23].

Proof. 1) $\Rightarrow$ : By Def. 1.1 and by Th.3.3.

$2) \Leftarrow$ : Firstly we prove the following statements:

$\stackrel{\circ}{1}$ For every $x_{1}^{m}, y_{1}^{m} \in Q^{m}$ and for every sequence $a_{1}^{n-m}$ over $Q$ the following implication holds

$A\left(x_{1}^{m}, a_{1}^{n-m}\right)=A\left(y_{1}^{m}, a_{1}^{n-m}\right) \Rightarrow x_{1}^{m}=y_{1}^{m}$.

$\stackrel{\circ}{2}(Q ; A)$ is an $(n, m)$-semigroup.

$\stackrel{\circ}{3}$ Law

$\left(3_{R}\right) A\left(b_{1}^{m}, a_{1}^{n-2 m},\left(a_{1}^{n-2 m}, b_{1}^{m}\right)^{-1}\right)=\mathbf{e}\left(a_{1}^{n-2 m}\right)$

holds in the algebra $\left(Q ; A,{ }^{-1}, \mathbf{e}\right)$.

$\stackrel{\circ}{4}$ Law

$\left(2_{R}\right) A\left(x_{1}^{m}, a_{1}^{n-2 m}, \mathbf{e}\left(a_{1}^{n-2 m}\right)\right)=x_{1}^{m}$

holds in the algebra $\left(Q ; A,{ }^{-1}, \mathbf{e}\right)$.

$\stackrel{\circ}{5}$ Law

$\left(3_{L}\right) \quad A\left(\left(a_{1}^{n-2 m}, b_{1}^{m}\right)^{-1}, a_{1}^{n-2 m}, b_{1}^{m}\right)=\mathbf{e}\left(a_{1}^{n-2 m}\right)$

holds in the algebra $\left(Q ; A,{ }^{-1}, \mathbf{e}\right)$.

${ }^{6}$ Law $\left(4_{L}\right)$ holds in the algebra $\left(Q ; A,{ }^{-1}, \mathbf{e}\right)$. 
Sketch of the proof of 1 :

$$
\begin{aligned}
& A\left(x_{1}^{m}, a_{1}^{n-2 m}, b_{1}^{m}\right)=A\left(y_{1}^{m}, a_{1}^{n-2 m}, b_{1}^{m}\right) \Rightarrow \\
& A\left(A\left(x_{1}^{m}, a_{1}^{n-2 m}, b_{1}^{m}\right), a_{1}^{n-2 m},\left(a_{1}^{n-2 m}, b_{1}^{m}\right)^{-1}\right)= \\
& A\left(A\left(y_{1}^{m}, a_{1}^{n-2 m}, b_{1}^{m}\right), a_{1}^{n-2 m},\left(a_{1}^{n-2 m}, b_{1}^{m}\right)^{-1}\right) \stackrel{\left(4_{R}\right)}{\Longrightarrow} x_{1}^{m}=y_{1}^{m} .
\end{aligned}
$$

Sketch of the proof of $\stackrel{\circ}{2}$ : By $\left(1_{L}\right), \stackrel{\circ}{1}$ and by Prop. $4.2_{1}$.

Sketch of the proof of $\stackrel{\circ}{3}$ :

$$
\begin{aligned}
& A\left(A\left(\mathbf{e}\left(a_{1}^{n-2 m}\right), a_{1}^{n-2 m}, b_{1}^{m}\right), a_{1}^{n-2 m},\left(a_{1}^{n-2 m}, b_{1}^{m}\right)^{-1}\right) \stackrel{\left(4_{R}\right)}{=} \mathbf{e}\left(a_{1}^{n-2 m}\right) \stackrel{\left(2_{L}\right)}{=} \\
& A\left(b_{1}^{m}, a_{1}^{n-2 m},\left(a_{1}^{n-2 m}, b_{1}^{m}\right)^{-1}\right)=\mathbf{e}\left(a_{1}^{n-2 m}\right) .
\end{aligned}
$$

Sketch of the proof of $\stackrel{\circ}{4}$ :

$$
\begin{aligned}
& A\left(x_{1}^{m}, a_{1}^{n-2 m}, \mathbf{e}\left(a_{1}^{n-2 m}\right)\right)=y_{1}^{m} \Rightarrow \\
& A\left(A\left(x_{1}^{m}, a_{1}^{n-2 m}, \mathbf{e}\left(a_{1}^{n-2 m}\right)\right), a_{1}^{n-2 m}, b_{1}^{m}\right)= \\
& A\left(y_{1}^{m}, a_{1}^{n-2 m}, b_{1}^{m}\right) \stackrel{\stackrel{\circ}{\rightleftharpoons}}{\Longrightarrow} 3 \\
& A\left(x_{1}^{m}, a_{1}^{n-2 m}, A\left(\mathbf{e}\left(a_{1}^{n-2 m}\right), a_{1}^{n-2 m}, b_{1}^{m}\right)\right)= \\
& A\left(y_{1}^{m}, a_{1}^{n-2 m}, b_{1}^{m}\right) \stackrel{\left(2_{L}\right)}{\Longrightarrow} \\
& A\left(x_{1}^{m}, a_{1}^{n-2 m}, b_{1}^{m}\right)= \\
& A\left(y_{1}^{m}, a_{1}^{n-2 m}, b_{1}^{m}\right) \stackrel{\stackrel{\circ}{\Longrightarrow}}{\Longrightarrow} x_{1}^{m}=y_{1}^{m} .
\end{aligned}
$$

Sketch of the proof of $\stackrel{\circ}{5}$ :

$$
\begin{aligned}
& A\left(\left(a_{1}^{n-2 m}, b_{1}^{m}\right)^{-1}, a_{1}^{n-2 m}, b_{1}^{m}\right)=y_{1}^{m} \Rightarrow \\
& A\left(A\left(\left(a_{1}^{n-2 m}, b_{1}^{m}\right)^{-1}, a_{1}^{n-2 m}, b_{1}^{m}\right), a_{1}^{n-2 m},\left(a_{1}^{n-2 m}, b_{1}^{m}\right)^{-1}\right)= \\
& A\left(y_{1}^{m}, a_{1}^{n-2 m},\left(a_{1}^{n-2 m}, b_{1}^{m}\right)^{-1}\right) \stackrel{\stackrel{\circ}{\Longrightarrow}}{\Longrightarrow}
\end{aligned}
$$

${ }^{3} A\left(A\left(x_{1}^{n}\right), x_{n+1}^{2 n-m}\right)=A\left(x_{1}^{n-m}, A\left(x_{n-m+1}^{2 n-m}\right)\right) ;(1 \mathrm{M})$ in Th. $5.4_{1}$.

${ }^{4}$ See footnote 3$)$. 


$$
\begin{aligned}
& A\left(\left(a_{1}^{n-2 m}, b_{1}^{m}\right)^{-1}, a_{1}^{n-2 m}, A\left(b_{1}^{m}, a_{1}^{n-2 m},\left(a_{1}^{n-2 m}, b_{1}^{m}\right)^{-1}\right)\right)= \\
& A\left(y_{1}^{m}, a_{1}^{n-2 m},\left(a_{1}^{n-2 m}, b_{1}^{m}\right)^{-1}\right) \stackrel{\stackrel{\circ}{\Longrightarrow}}{\Longrightarrow} \\
& A\left(\left(a_{1}^{n-2 m}, b_{1}^{m}\right)^{-1}, a_{1}^{n-2 m}, \mathbf{e}\left(a_{1}^{n-2 m}\right)\right)= \\
& A\left(y_{1}^{m}, a_{1}^{n-2 m},\left(a_{1}^{n-2 m}, b_{1}^{m}\right)^{-1}\right) \stackrel{\stackrel{\circ}{4,(2 L)}}{\Longrightarrow} \\
& A\left(\mathbf{e}\left(a_{1}^{n-2 m}\right), a_{1}^{n-2 m},\left(a_{1}^{n-2 m}, b_{1}^{m}\right)^{-1}\right)= \\
& A\left(y_{1}^{m}, a_{1}^{n-2 m},\left(a_{1}^{n-2 m}, b_{1}^{m}\right)^{-1}\right) \stackrel{\stackrel{\circ}{\Longrightarrow}}{\Longrightarrow} y_{1}^{m}=\mathbf{e}\left(a_{1}^{n-2 m}\right) \text {. }
\end{aligned}
$$

Sketch of the proof of $\stackrel{\circ}{6}$ :

$$
\begin{aligned}
& A\left(\left(a_{1}^{n-2 m}, b_{1}^{m}\right)^{-1}, a_{1}^{n-2 m}, A\left(b_{1}^{m}, a_{1}^{n-2 m}, x_{1}^{m}\right)\right)_{\stackrel{\circ}{\stackrel{\circ}{~}}} 5 \\
& A\left(A\left(\left(a_{1}^{n-2 m}, b_{1}^{m}\right)^{-1}, a_{1}^{n-2 m}, b_{1}^{m}\right), a_{1}^{n-2 m}, x_{1}^{m}\right) \stackrel{\circ}{=} \\
& A\left(\mathbf{e}\left(a_{1}^{n-2 m}\right), a_{1}^{n-2 m}, x_{1}^{m}\right)^{\left(2_{L}\right)}=x_{1}^{m} .
\end{aligned}
$$

Finally, by $\left(1_{L}\right),\left(4_{R}\right), \stackrel{\circ}{6}$ and by Theorem $5.2_{1}$, we conclude that $(Q ; A)$ is an $(n, m)$-group. Whence, by " $\Rightarrow "$, we obtain Th. $5 \cdot 3_{1}$.

Similarly, it is posible to prove also the following proposition:

$5.3_{2}$. Theorem [19]: Let $n \geq 2 m, m \geq 2$ and let $(Q ; A)$ be an $(n, m)$-groupoid. Then, $(Q ; A)$ is an $(n, m)$-group iff there are mappings ${ }^{-1}$ and $\mathbf{e}$, respectively, of the sets $Q^{n-m}$ and $Q^{n-2 m}$ into the set $Q^{m}$ such that the laws

$\left(1_{R}\right) A\left(x_{1}^{n-m-1}, A\left(x_{n-m}^{2 n-m-1}\right), x_{2 n-m}\right)=A\left(x_{1}^{n-m}, A\left(x_{n-m+1}^{2 n-m}\right)\right)$,

$\left(2_{R}\right) A\left(x_{1}^{m}, a_{1}^{n-2 m}, \mathbf{e}\left(a_{1}^{n-2 m}\right)\right)=x_{1}^{m}$ and

(4 $\left.4_{L}\right) A\left(\left(a_{1}^{n-2 m}, b_{1}^{m}\right)^{-1}, a_{1}^{n-2 m}, A\left(b_{1}^{m}, a_{1}^{n-2 m}, x_{1}^{m}\right)\right)=x_{1}^{m}$

hold in the algebra $\left(Q ; A,{ }^{-1}, \mathbf{e}\right)$.

Remark: For $m=1$ Th. $5.3_{2}$ is proved in [17]. Cf. III-3 in [23].

5.41. Theorem [19]: Let $n \geq 2 m, m \geq 2$ and let $(Q ; A)$ be an $(n, m)$-groupoid. Then, $(Q ; A)$ is $(n, m)$-group iff there are mappings ${ }^{-1}$ and $\mathbf{e}$, respectively, of the sets $Q^{n-m}$ and $Q^{n-2 m}$ into the set $Q^{m}$ such that the laws

$$
\begin{aligned}
& \left(1_{L}\right) A\left(A\left(x_{1}^{n}\right), x_{n+1}^{2 n-m}\right)=A\left(x_{1}, A\left(x_{2}^{n+1}\right), x_{n+2}^{2 n-m}\right), \\
& \left(1_{M}\right) A\left(A\left(x_{1}^{n}\right), x_{n+1}^{2 n-m}\right)=A\left(x_{1}^{n-m}, A\left(x_{n-m+1}^{2 n-m}\right)\right), \\
& \left(2_{R}\right) A\left(x_{1}^{m}, a_{1}^{n-2 m}, \mathbf{e}\left(a_{1}^{n-2 m}\right)\right)=x_{1}^{m} \text { and }
\end{aligned}
$$

\footnotetext{
${ }^{5}$ See footnote 3$)$.
} 
$\left(3_{R}\right) A\left(b_{1}^{m}, a_{1}^{n-2 m},\left(a_{1}^{n-2 m}, b_{1}^{m}\right)^{-1}\right)=\mathbf{e}\left(a_{1}^{n-2 m}\right)$

hold in the algebra $\left(Q ; A,{ }^{-1}, \mathbf{e}\right)$.

Remark: The case $m=1$ was described in [17]. Cf. III-3 in [23].

Proof. 1) $\Rightarrow$ : By Def. 1.1 and by Th. 3.3.

2) $\Leftarrow$ : Firstly we prove the following statements:

$\widehat{1}$ For every $x_{1}^{m}, y_{1}^{m} \in Q^{m}$ and for every sequence $a_{1}^{n-m}$ over $Q$ the following implication holds

$A\left(x_{1}^{m}, a_{1}^{n-m}\right)=A\left(y_{1}^{m}, a_{1}^{n-m}\right) \Rightarrow x_{1}^{m}=y_{1}^{m}$.

$\widehat{2}$ Law

$$
A\left(\mathbf{e}\left(a_{1}^{n-2 m}\right), a_{1}^{n-2 m}, x_{1}^{m}\right)=x_{1}^{m}
$$

hold in the algebra $\left(Q ; A,{ }^{-1}, \mathbf{e}\right)$.

$\widehat{3}$ Law

$$
A\left(\left(a_{1}^{n-2 m}, b_{1}^{m}\right)^{-1}, a_{1}^{n-2 m}, b_{1}^{m}\right)=\mathbf{e}\left(a_{1}^{n-2 m}\right)
$$

hold in the algebra $\left(Q ; A,{ }^{-1}, \mathbf{e}\right)$.

$\widehat{4}$ Laws $\left(4_{L}\right)$ and $\left(4_{R}\right)$ hold in the algebra $\left(Q ; A,{ }^{-1}, \mathbf{e}\right)$.

Sketch of the proof of $\widehat{1}$ :

$$
\begin{aligned}
& A\left(x_{1}^{m}, a_{1}^{n-2 m}, b_{1}^{m}\right)=A\left(y_{1}^{m}, a_{1}^{n-2 m}, b_{1}^{m}\right) \Rightarrow \\
& A\left(A\left(x_{1}^{m}, a_{1}^{n-2 m}, b_{1}^{m}\right), a_{1}^{n-2 m},\left(a_{1}^{n-2 m}, b_{1}^{m}\right)^{-1}\right)= \\
& A\left(A\left(y_{1}^{m}, a_{1}^{n-2 m}, b_{1}^{m}\right), a_{1}^{n-2 m},\left(a_{1}^{n-2 m}, b_{1}^{m}\right)^{-1}\right) \stackrel{\left(1_{M}\right)}{\Rightarrow} \\
& A\left(x_{1}^{m}, a_{1}^{n-2 m}, A\left(b_{1}^{m}, a_{1}^{n-2 m},\left(a_{1}^{n-2 m}, b_{1}^{m}\right)^{-1}\right)\right)= \\
& A\left(y_{1}^{m}, a_{1}^{n-2 m}, A\left(b_{1}^{m}, a_{1}^{n-2 m},\left(a_{1}^{n-2 m}, b_{1}^{m}\right)^{-1}\right)\right) \stackrel{\left(3_{R}\right)}{\Rightarrow} \\
& A\left(x_{1}^{m}, a_{1}^{n-2 m}, \mathbf{e}\left(a_{1}^{n-2 m}\right)\right)= \\
& A\left(y_{1}^{m}, a_{1}^{n-2 m}, \mathbf{e}\left(a_{1}^{n-2 m}\right)\right) \stackrel{\left(2_{R}\right)}{\Rightarrow} x_{1}^{m}=y_{1}^{m} .
\end{aligned}
$$

Sketch of the proof of $\widehat{2}$ :

$$
\begin{aligned}
& A\left(\mathbf{e}\left(a_{1}^{n-2 m}\right), a_{1}^{n-2 m}, x_{1}^{m}\right)=y_{1}^{m} \Rightarrow \\
& A\left(A\left(\mathbf{e}\left(a_{1}^{n-2 m}\right), a_{1}^{n-2 m}, x_{1}^{m}\right), a_{1}^{n-2 m},\left(a_{1}^{n-2 m}, x_{1}^{m}\right)^{-1}\right)= \\
& A\left(y_{1}^{m}, a_{1}^{n-2 m}, a_{1}^{n-2 m},\left(a_{1}^{n-2 m}, x_{1}^{m}\right)^{-1}\right)^{\left(1_{M}\right)} \Rightarrow \\
& A\left(\mathbf{e}\left(a_{1}^{n-2 m}\right), a_{1}^{n-2 m}, A\left(x_{1}^{m}, a_{1}^{n-2 m},\left(a_{1}^{n-2 m}, x_{1}^{m}\right)^{-1}\right)\right)= \\
& A\left(y_{1}^{m}, a_{1}^{n-2 m},\left(a_{1}^{n-2 m}, x_{1}^{m}\right)^{-1}\right) \stackrel{\left(3_{R}\right)}{\Rightarrow}
\end{aligned}
$$




$$
\begin{aligned}
& A\left(\mathbf{e}\left(a_{1}^{n-2 m}\right), a_{1}^{n-2 m}, \mathbf{e}\left(a_{1}^{n-2 m}\right)\right)= \\
& A\left(y_{1}^{m}, a_{1}^{n-2 m},\left(a_{1}^{n-2 m}, x_{1}^{m}\right)^{-1}\right) \stackrel{\left(2_{R}\right)}{\Rightarrow} \\
& \mathbf{e}\left(a_{1}^{n-2 m}\right)=A\left(y_{1}^{m}, a_{1}^{n-2 m},\left(a_{1}^{n-2 m}, x_{1}^{m}\right)^{-1}\right) \stackrel{\left(3_{R}\right)}{\Rightarrow} \\
& A\left(x_{1}^{m}, a_{1}^{n-2 m},\left(a_{1}^{n-2 m}, x_{1}^{m}\right)^{-1}\right)= \\
& A\left(y_{1}^{m}, a_{1}^{n-2 m},\left(a_{1}^{n-2 m}, x_{1}^{m}\right)^{-1}\right) \stackrel{\widehat{1}}{\Rightarrow} x_{1}^{m}=y_{1}^{m} .
\end{aligned}
$$

Sketch of the proof of $\widehat{3}$ :

$$
\begin{aligned}
& A\left(\left(a_{1}^{n-2 m}, b_{1}^{m}\right)^{-1}, a_{1}^{n-2 m}, b_{1}^{m}\right)=y_{1}^{m} \Rightarrow \\
& A\left(A\left(\left(a_{1}^{n-2 m}, b_{1}^{m}\right)^{-1}, a_{1}^{n-2 m}, b_{1}^{m}\right), a_{1}^{n-2 m},\left(a_{1}^{n-2 m}, b_{1}^{m}\right)^{-1}\right)= \\
& A\left(y_{1}^{m}, a_{1}^{n-2 m},\left(a_{1}^{n-2 m}, b_{1}^{m}\right)^{-1}\right)^{\left(1_{M}\right)} \Rightarrow \\
& A\left(\left(a_{1}^{n-2 m}, b_{1}^{m}\right)^{-1}, a_{1}^{n-2 m}, A\left(b_{1}^{m}, a_{1}^{n-2 m},\left(a_{1}^{n-2 m}, b_{1}^{m}\right)^{-1}\right)\right)= \\
& A\left(y_{1}^{m}, a_{1}^{n-2 m},\left(a_{1}^{n-2 m}, b_{1}^{m}\right)^{-1}\right)^{\left(3_{R}\right)} \Rightarrow \\
& \left.A\left(a_{1}^{n-2 m}, b_{1}^{m}\right)^{-1}, a_{1}^{n-2 m}, \mathbf{e}\left(a_{1}^{n-2 m}\right)\right)= \\
& A\left(y_{1}^{m}, a_{1}^{n-2 m},\left(a_{1}^{n-2 m}, b_{1}^{m}\right)^{-1}\right) \stackrel{\left(2_{R}\right)^{\widehat{2}}}{\Longrightarrow} \\
& A\left(\mathbf{e}\left(a_{1}^{n-2 m}\right), a_{1}^{n-2 m},\left(a_{1}^{n-2 m}, x_{1}^{m}\right)^{-1}\right)= \\
& A\left(y_{1}^{m}, a_{1}^{n-2 m},\left(a_{1}^{n-2 m}, b_{1}^{m}\right)^{-1}\right) \stackrel{\widehat{1}}{\Longrightarrow} y_{1}^{m}=\mathbf{e}\left(a_{1}^{n-2 m}\right) .
\end{aligned}
$$

Sketch of the proof of $\widehat{4}$ :

a) $A\left(\left(a_{1}^{n-2 m}, b_{1}^{m}\right)^{-1}, a_{1}^{n-2 m}, A\left(b_{1}^{m}, a_{1}^{n-2 m}, x_{1}^{m}\right)^{\left(1_{M}\right)}\right.$

$$
\begin{aligned}
& A\left(A\left(\left(a_{1}^{n-2 m}, b_{1}^{m}\right)^{-1}, a_{1}^{n-2 m}, b_{1}^{m}\right), a_{1}^{n-2 m}, x_{1}^{m}\right) \stackrel{\widehat{3}}{=} \\
& A\left(\mathbf{e}\left(a_{1}^{n-2 m}\right), a_{1}^{n-2 m}, x_{1}^{m}\right) \stackrel{\widehat{(2)}}{=} x_{1}^{m} .
\end{aligned}
$$

b) $\left.A\left(A\left(x_{1}^{m}, a_{1}^{n-2 m}, b_{1}^{m}\right), a_{1}^{n-2 m},\left(a_{1}^{n-2 m}, b_{1}^{m}\right)^{-1}\right)^{\left(1_{M}\right.}\right)$

$$
\begin{aligned}
& A\left(x_{1}^{m}, a_{1}^{n-2 m}, A\left(b_{1}^{m}, a_{1}^{n-2 m},\left(a_{1}^{n-2 m}, b_{1}^{m}\right)^{-1}\right) \stackrel{\left(3_{R}\right)}{=}\right. \\
& A\left(x_{1}^{m}, a_{1}^{n-2 m}, \mathbf{e}\left(a_{1}^{n-2 m}\right) \stackrel{\left(2_{R}\right)}{=} x_{1}^{m} .\right.
\end{aligned}
$$

Finally, by $(1 L), \widehat{4}$ and by Th. $5.1_{1}$, we conclude that $(Q ; A)$ is an $(n, m)$-group. Whence, by " $\Rightarrow "$, we obtain Th. 5.4 .

Similarly, it is posible to proved also the following proposition: 
5.42. Theorem [19]: Let $n \geq 2 m, m \geq 2$ and let $(Q ; A)$ be an $(n, m)$-groupoid. Then, $(Q ; A)$ is an $(n, m)$-group iff there are mappings ${ }^{-1}$ and $\mathbf{e}$, respectively, of the sets $Q^{n-m}$ and $Q^{n-2 m}$ into the set $Q^{m}$ such that the laws

$\left(1_{R}\right) A\left(x_{1}^{n-m-1}, A\left(x_{n-m}^{2 n-m-1}\right), x_{2 n-m}\right)=A\left(x_{1}^{n-m}, A\left(x_{n-m+1}^{2 n-m}\right)\right)$,

(1 $\left.1_{M}\right) A\left(A\left(x_{1}^{n}\right), x_{n+1}^{2 n-m}\right)=A\left(x_{1}^{n-m}, A\left(x_{n-m+1}^{2 n-m}\right)\right)$,

$\left(2_{L}\right) \quad A\left(\mathbf{e}\left(a_{1}^{n-2 m}\right), a_{1}^{n-2 m}, x_{1}^{m}\right)=x_{1}^{m}$ and

$\left(3_{L}\right) \quad A\left(\left(a_{1}^{n-2 m}, b_{1}^{m}\right)^{-1}, a_{1}^{n-2 m}, b_{1}^{m}\right)=\mathbf{e}\left(a_{1}^{n-2 m}\right)$

hold in the algebra $\left(Q ; A,{ }^{-1}, \mathbf{e}\right)$.

Remark: The case $m=1$ was described in [17]. Cf. III-3 in [23].

5.5. Theorem [26]: Let $n \geq 3 m, m \geq 2$ and let $(Q ; A)$ be an $(n, m)$-groupoid. Then, $(Q ; A)$ is an $(n, m)$-group iff there is a mapping e of the set $Q^{n-2 m}$ into the set $Q^{m}$ such that the laws

$\left(1_{L}\right) \quad A\left(A\left(x_{1}^{n}\right), x_{n+1}^{2 n-m}\right)=A\left(x_{1}, A\left(x_{2}^{n+1}\right), x_{n+2}^{2 n-m}\right)$,

$\left(1_{L m}\right) A\left(A\left(a_{1}^{m}, b_{1}^{n-m}\right), c_{1}^{m}, d_{1}^{n-2 m}\right)=A\left(a_{1}^{m}, A\left(b_{1}^{n-m}, c_{1}^{m}\right), d_{1}^{n-2 m}\right)$,

$\left(2_{L}\right) \quad A\left(\mathbf{e}\left(a_{1}^{n-2 m}\right), a_{1}^{n-2 m}, x_{1}^{m}\right)=x_{1}^{m}$ and

$\left(2_{R}\right) \quad A\left(x_{1}^{m}, a_{1}^{n-2 m}, \mathbf{e}\left(a_{1}^{n-2 m}\right)\right)=x_{1}^{m}$

hold in the algebra $(Q ; A, \mathbf{e})$.

Remarks: a) For $m=1$ : $(1 L)=(1 L m)$. b) For $m=1$ Th. $5.5_{1}$ is proved in [17].

Cf. IX-2 in [23].

Proof. 1) $\Rightarrow$ : By Def. 1.1 and by Th. 3.3.

$2) \Leftarrow$ : Firstly we prove the following statements:

$\overline{1}$ For every $x_{1}^{m}, y_{1}^{m}, b_{1}^{m} \in Q^{m}$ and for every sequence $a_{1}^{n-2 m}$ over $Q$ the following implication holds

$A\left(x_{1}^{m}, b_{1}^{m}, a_{1}^{n-2 m}\right)=A\left(y_{1}^{m}, b_{1}^{m}, a_{1}^{n-2 m}\right) \Rightarrow x_{1}^{m}=y_{1}^{m}$.

$\overline{2}(Q ; A)$ is an $(n, m)$-semigroup.

$\overline{3}$ For every $x_{1}^{m}, y_{1}^{m}, b_{1}^{m} \in Q^{m}$ and for every sequence $a_{1}^{n-2 m}$ over $Q$ the following implication holds

$A\left(a_{1}^{n-m}, b_{1}^{m}, x_{1}^{m}\right)=A\left(a_{1}^{n-m}, b_{1}^{m}, y_{1}^{m}\right) \Rightarrow x_{1}^{m}=y_{1}^{m}$.

$\overline{4}$ For every $a_{1}^{n} \in Q$ there is exactly one sequence $x_{1}^{m}$ over $Q$ and exactly one sequence $y_{1}^{m}$ over $Q$ such that the following equalities hold

$A\left(a_{1}^{n-m}, x_{1}^{m}\right)=a_{n-m+1}^{n}$ and $A\left(y_{1}^{m}, a_{1}^{n-m}\right)=a_{n-m+1}^{n}$.

Sketch of the proof of $\overline{1}$ :

$A\left(x_{1}^{m}, b_{1}^{m}, a_{1}^{n-2 m}\right)=A\left(y_{1}^{m}, b_{1}^{m}, a_{1}^{n-2 m}\right) \stackrel{n \geq 3 m}{\Longrightarrow}$ 
$A\left(A\left(x_{1}^{m}, b_{1}^{m}, a_{1}^{n-2 m}\right), \mathbf{e}\left(a_{1}^{n-2 m}\right), c_{1}^{n-3 m}, \mathbf{e}\left(b_{1}^{m}, c_{1}^{n-3 m}\right)\right)=$ $A\left(A\left(y_{1}^{m}, b_{1}^{m}, a_{1}^{n-2 m}\right), \mathbf{e}\left(a_{1}^{n-2 m}\right), c_{1}^{n-3 m}, \mathbf{e}\left(b_{1}^{m}, c_{1}^{n-3 m}\right)\right) \stackrel{(1 L m)}{\Longrightarrow}$

$A\left(x_{1}^{m}, A\left(b_{1}^{m}, a_{1}^{n-2 m}, \mathbf{e}\left(a_{1}^{n-2 m}\right)\right), c_{1}^{n-3 m}, \mathbf{e}\left(b_{1}^{m}, c_{1}^{n-3 m}\right)\right)=$

$A\left(y_{1}^{m}, A\left(b_{1}^{m}, a_{1}^{n-2 m}, \mathbf{e}\left(a_{1}^{n-2 m}\right)\right), c_{1}^{n-3 m}, \mathbf{e}\left(b_{1}^{m}, c_{1}^{n-3 m}\right)\right) \stackrel{(2 R)}{\Longrightarrow}$

$A\left(x_{1}^{m}, b_{1}^{m}, c_{1}^{n-3 m}, \mathbf{e}\left(b_{1}^{m}, c_{1}^{n-3 m}\right)\right)=$

$A\left(x_{1}^{m}, b_{1}^{m}, c_{1}^{n-3 m}, \mathbf{e}\left(b_{1}^{m}, c_{1}^{n-3 m}\right)\right) \stackrel{(2 R)}{\Longrightarrow} x_{1}^{m}=y_{1}^{m}$.

The proof of $\overline{2}$ : By $\overline{1},\left(1_{L}\right)$ and by Prop. $4.2_{1}$.

Sketch of the proof of $\overline{3}$ :

$$
\begin{aligned}
& A\left(a_{1}^{n-2 m}, b_{1}^{m}, x_{1}^{m}\right)=A\left(a_{1}^{n-2 m}, b_{1}^{m}, y_{1}^{m}\right)^{n \geq 3 m} \stackrel{\underline{\Longrightarrow}}{ } A\left(\mathbf{e}\left(c_{1}^{n-3 m}, b_{1}^{m}\right), c_{1}^{n-3 m}, \mathbf{e}\left(a_{1}^{n-2 m}\right), A\left(a_{1}^{n-2 m}, b_{1}^{m}, x_{1}^{m}\right)\right)= \\
& A\left(\mathbf{e}\left(c_{1}^{n-3 m}, b_{1}^{m}\right), c_{1}^{n-3 m}, \mathbf{e}\left(a_{1}^{n-2 m}\right), A\left(a_{1}^{n-2 m}, b_{1}^{m}, y_{1}^{m}\right)\right) \stackrel{\overline{2}}{\Longrightarrow} \\
& \left.A\left(\mathbf{e}\left(c_{1}^{n-3 m}, b_{1}^{m}\right), c_{1}^{n-3 m}, A\left(\mathbf{e}\left(a_{1}^{n-2 m}\right), a_{1}^{n-2 m}, b_{1}^{m}\right), x_{1}^{m}\right)\right)= \\
& \left.A\left(\mathbf{e}\left(c_{1}^{n-3 m}, b_{1}^{m}\right), c_{1}^{n-3 m}, A\left(\mathbf{e}\left(a_{1}^{n-2 m}\right), a_{1}^{n-2 m}, b_{1}^{m}\right), y_{1}^{m}\right)\right) \stackrel{(2 L)}{\Longrightarrow} \\
& A\left(\mathbf{e}\left(c_{1}^{n-3 m}, b_{1}^{m}\right), c_{1}^{n-3 m}, b_{1}^{m}, x_{1}^{m}\right)= \\
& A\left(\mathbf{e}\left(c_{1}^{n-3 m}, b_{1}^{m}\right), c_{1}^{n-3 m}, b_{1}^{m}, y_{1}^{m}\right) \stackrel{(2 L)}{\Longrightarrow} x_{1}^{m}=y_{1}^{m} .
\end{aligned}
$$

Sketch of the proof of $\overline{4}$ :

a) $A\left(a_{1}^{n-2 m}, b_{1}^{m}, x_{1}^{m}\right)=d_{1}^{m} \stackrel{\overline{3}}{\Longleftrightarrow} 6$

$A\left(\mathbf{e}\left(c_{1}^{n-3 m}, b_{1}^{m}\right), c_{1}^{n-3 m}, \mathbf{e}\left(a_{1}^{n-2 m}\right), A\left(a_{1}^{n-2 m}, b_{1}^{m}, x_{1}^{m}\right)\right)=$

$A\left(\mathbf{e}\left(c_{1}^{n-3 m}, b_{1}^{m}\right), c_{1}^{n-3 m}, \mathbf{e}\left(a_{1}^{n-2 m}\right), d_{1}^{m}\right) \stackrel{{ }^{2},(2 L)}{\Longleftrightarrow}$

$x_{1}^{m}=A\left(\mathbf{e}\left(c_{1}^{n-3 m}, b_{1}^{m}\right), c_{1}^{n-3 m}, \mathbf{e}\left(a_{1}^{n-2 m}\right), d_{1}^{m}\right)$.

b) $A\left(y_{1}^{m}, b_{1}^{m}, a_{1}^{n-2 m}\right)=d_{1}^{m} \stackrel{\overline{1}}{\Longleftrightarrow}$

$A\left(A\left(y_{1}^{m}, b_{1}^{m}, a_{1}^{n-2 m}\right), \mathbf{e}\left(a_{1}^{n-2 m}\right), c_{1}^{n-3 m}, \mathbf{e}\left(b_{1}^{m}, c_{1}^{n-3 m}\right)\right)=$

$A\left(d_{1}^{m}, \mathbf{e}\left(a_{1}^{n-2 m}\right), c_{1}^{n-3 m}, \mathbf{e}\left(b_{1}^{m}, c_{1}^{n-3 m}\right)\right) \stackrel{\overline{2},(2 R)}{\Longleftrightarrow}$

$y_{1}^{m}=A\left(d_{1}^{m}, \mathbf{e}\left(a_{1}^{n-2 m}\right), c_{1}^{n-3 m}, \mathbf{e}\left(b_{1}^{m}, c_{1}^{n-3 m}\right)\right)$.

c) By $a$ ) and $\overline{1}$ and by $b$ ) and $\overline{3}$, we obtain $\overline{4}$.

Finally, by $\overline{2}, \overline{4}$ and by Prop. 4.1 , we conclude that $(Q ; A)$ is an $(n, m)$-group. Whence, by" $\Rightarrow "$, we obtain Th $5.5_{1}$.

Similarly, one could prove also the following proposition:

5.5. . Theorem [26]: Let $n \geq 3 m, m \geq 2$ and let $(Q ; A)$ be an $(n, m)$-groupoid. Then, $(Q ; A)$ is an $(n, m)$-group iff there is a mapping e of the set $Q^{n-2 m}$ into the set $Q^{m}$ such that the laws

$6 \stackrel{\overline{3}}{\rightleftarrows} . \Rightarrow:$ monotony. 
$\left(1_{R}\right) \quad A\left(x_{1}^{n-m-1}, A\left(x_{n-m}^{2 n-m-1}\right), x_{2 n-m}\right)=A\left(x_{1}^{n-m}, A\left(x_{n-m+1}^{2 n-m}\right)\right)$,

$\left(1_{R m}\right) \quad A\left(a_{1}^{n-2 m}, A\left(b_{1}^{m}, c_{1}^{n-m}\right), d_{1}^{m}\right)=A\left(a_{1}^{n-2 m}, b_{1}^{m}, A\left(c_{1}^{n-m}, d_{1}^{m}\right)\right)$,

$\left(2_{L}\right) \quad A\left(\mathbf{e}\left(a_{1}^{n-2 m}\right), a_{1}^{n-2 m}, x_{1}^{m}\right)=x_{1}^{m}$ and

$\left(2_{R}\right) \quad A\left(x_{1}^{m}, a_{1}^{n-2 m}, \mathbf{e}\left(a_{1}^{n-2 m}\right)\right)=x_{1}^{m}$

hold in the algebra $(Q ; A, \mathbf{e})$.

Remarks: a) For $m=1:(1 R)=(1 R m)$. b) For $m=1$ Th. $5.5_{2}$ is proved in [17].

Cf. IX-2 in [23].

5.61. Theorem [19]: Let $n \geq 3 m, m \geq 2$ and let $(Q ; A)$ be an $(n, m)$-groupoid.

Then, $(Q ; A)$ is an $(n, m)$-group iff there are mappings ${ }^{-1}$ and $\mathbf{e}$, respectively, of the sets $Q^{n-m}$ and $Q^{n-2 m}$ into the set $Q^{m}$ such that the laws

$\left(1_{L}\right) A\left(A\left(x_{1}^{n}\right), x_{n+1}^{2 n-m}\right)=A\left(x_{1}, A\left(x_{2}^{n+1}\right), x_{n+2}^{2 n-m}\right)$,

$\left(1_{L m}\right) A\left(A\left(a_{1}^{m}, b_{1}^{n-m}\right), c_{1}^{m}, d_{1}^{n-2 m}\right)=A\left(a_{1}^{m}, A\left(b_{1}^{n-m}, c_{1}^{m}\right), d_{1}^{n-2 m}\right)$,

$\left(2_{R}\right) A\left(x_{1}^{m}, a_{1}^{n-2 m}, \mathbf{e}\left(a_{1}^{n-2 m}\right)\right)=x_{1}^{m}$

$\left(3_{R}\right) A\left(b_{1}^{m}, a_{1}^{n-2 m},\left(a_{1}^{n-2 m}, b_{1}^{m}\right)^{-1}\right)=\mathbf{e}\left(a_{1}^{n-2 m}\right)$

hold in the algebra $\left(Q ; A,{ }^{-1}, \mathbf{e}\right)$.

Remarks: a) For $m=1:(1 L m)=(1 L)$. b) For $m=1$ see III-3 in [23].

Proof. 1) $\Rightarrow$ : By Def. 1.1 and by Th. 3.3.

$2) \Leftarrow$ : Firstly we prove the following statements:

$\overline{\overline{1}}$ For every $x_{1}^{m}, y_{1}^{m}, b_{1}^{m} \in Q^{m}$ and for every sequence $a_{1}^{n-2 m}$ over $Q$ the following implication holds

$A\left(x_{1}^{m}, b_{1}^{m}, a_{1}^{n-2 m}\right)=A\left(y_{1}^{m}, b_{1}^{m}, a_{1}^{n-2 m}\right) \Rightarrow x_{1}^{m}=y_{1}^{m}$.

$\overline{\overline{2}}(Q ; A)$ is an $(n, m)$-semigroup.

$\overline{\overline{3}} \mathrm{Law}$

$\left(2_{L}\right) \quad A\left(\mathbf{e}\left(a_{1}^{n-2 m}\right), a_{1}^{n-2 m}, x_{1}^{m}\right)=x_{1}^{m}$

hold in the algebra $\left(Q ; A,{ }^{-1}, \mathbf{e}\right)$.

Sketch of the proof of $\overline{\overline{1}}$ : Sketch of the proof of $\overline{1}$ in the proof of Th. $5.5_{1}$.

The proof of $\overline{\overline{2}}$ : By $\overline{\overline{1}},(1 L)$ and by Prop. $4.2_{1}$.

Sketch of the proof of $\overline{\overline{3}}$ :

$$
\begin{aligned}
& A\left(\mathbf{e}\left(a_{1}^{n-2 m}\right), a_{1}^{n-2 m}, x_{1}^{m}\right)=y_{1}^{m} \Rightarrow \\
& A\left(A\left(\mathbf{e}\left(a_{1}^{n-2 m}\right), a_{1}^{n-2 m}, x_{1}^{m}\right), a_{1}^{n-2 m},\left(a_{1}^{n-2 m}, x_{1}^{m}\right)^{-1}\right)= \\
& A\left(y_{1}^{m}, a_{1}^{n-2 m},\left(a_{1}^{n-2 m}, x_{1}^{m}\right)^{-1}\right) \stackrel{\overline{2}}{\Longrightarrow} \\
& A\left(\mathbf{e}\left(a_{1}^{n-2 m}\right), a_{1}^{n-2 m}, A\left(x_{1}^{m}, a_{1}^{n-2 m},\left(a_{1}^{n-2 m}, x_{1}^{m}\right)^{-1}\right)\right)= \\
& A\left(y_{1}^{m}, a_{1}^{n-2 m},\left(a_{1}^{n-2 m}, x_{1}^{m}\right)^{-1}\right) \stackrel{(3 R)}{\Longrightarrow}
\end{aligned}
$$


$A\left(\mathbf{e}\left(a_{1}^{n-2 m}\right), a_{1}^{n-2 m}, \mathbf{e}\left(a_{1}^{n-2 m}\right)\right)=$

$A\left(y_{1}^{m}, a_{1}^{n-2 m},\left(a_{1}^{n-2 m}, x_{1}^{m}\right)^{-1}\right) \stackrel{(2 R)}{\Longrightarrow}$

$\mathbf{e}\left(a_{1}^{n-2 m}\right)=$

$A\left(y_{1}^{m}, a_{1}^{n-2 m},\left(a_{1}^{n-2 m}, x_{1}^{m}\right)^{-1}\right) \stackrel{(3 R)}{\Longrightarrow}$

$A\left(x_{1}^{m}, a_{1}^{n-2 m},\left(a_{1}^{n-2 m}, x_{1}^{m}\right)^{-1}\right)=$

$A\left(y_{1}^{m}, a_{1}^{n-2 m},\left(a_{1}^{n-2 m}, x_{1}^{m}\right)^{-1}\right) \stackrel{\overline{\overline{1}}}{\Longrightarrow} x_{1}^{m}=y_{1}^{m}$.

[Cf. the proof of Th. 5.4.]

Finally, by $(1 L),(1 L m),(2 R), \overline{\overline{3}}$ and by Th. $5.5_{1}$, we conclude that $(Q ; A)$ is an $(n, m)$-group. Whence, by " $\Rightarrow "$, we obtain Th. 5.6 .

Similarly, it is posible to prove also the following proposition:

5.6. Theorem [19]: Let $n \geq 3 m, m \geq 2$ and let $(Q ; A)$ be an $(n, m)$-groupoid. Then, $(Q ; A)$ is an $(n, m)$-group iff there are mappings ${ }^{-1}$ and $\mathbf{e}$, respectively, of the sets $Q^{n-m}$ and $Q^{n-2 m}$ into the set $Q^{m}$ such that the laws

$\left(1_{R}\right) \quad A\left(x_{1}^{n-m-1}, A\left(x_{n-m}^{2 n-m-1}\right), x_{2 n-m}\right)=A\left(x_{1}^{n-m}, A\left(x_{n-m+1}^{2 n-m}\right)\right)$,

$\left(1_{R m}\right) \quad A\left(a_{1}^{n-2 m}, A\left(b_{1}^{m}, c_{1}^{n-m}\right), d_{1}^{m}\right)=A\left(a_{1}^{n-2 m}, b_{1}^{m}, A\left(c_{1}^{n-m}, d_{1}^{m}\right)\right)$,

$\left(2_{L}\right) \quad A\left(\mathbf{e}\left(a_{1}^{n-2 m}\right), a_{1}^{n-2 m}, x_{1}^{m}\right)=x_{1}^{m}$ and

$\left(3_{L}\right) \quad A\left(\left(a_{1}^{n-2 m}, b_{1}^{m}\right)^{-1}, a_{1}^{n-2 m}, b_{1}^{m}\right)=\mathbf{e}\left(a_{1}^{n-2 m}\right)$

hold in the algebra $\left(Q ; A,{ }^{-1}, \mathbf{e}\right)$.

Remarks: a) For $m=1:(1 R m)=(1 R)$. b) For $m=1$ see III-3 in [23].

5.7. Theorem [22]: Let $n \geq 3 m, m \geq 2$ and let $(Q ; A)$ be an $(n, m)$-groupoid. Then, $(Q ; A)$ is an $(n, m)$-group iff there is $i \in\{m+1, \ldots, n-2 m+1\}$ such that the following statements hold:

(a) The $<i-1, i>-$ associative law holds in $(Q ; A)$;

(b) The $<i, i+1>-$ associative law holds in $(Q ; A)$; and

(c) For every $a_{1}^{n} \in Q$ there is exactly one $x_{1}^{m} \in Q^{m}$ such that the following equality holds

$$
A\left(a_{1}^{i-1}, x_{1}^{m}, a_{i}^{n-m}\right)=a_{n-m+1}^{n} .
$$

Remark: For $m=1$ Th. 5.7 is proved in [20]. Cf. IX-3 in [23].

Proof. 1) $(c) \Leftrightarrow\left(c_{1}\right) \wedge\left(c_{2}\right)$, where

$\left(c_{1}\right)$ For every $a_{1}^{n-m}, x_{1}^{m}, y_{1}^{m} \in Q$ the implication holds

$$
A\left(a_{1}^{i-1}, x_{1}^{m}, a_{i}^{n-m}\right)=A\left(a_{1}^{i-1}, y_{1}^{m}, a_{i}^{n-m}\right) \Rightarrow x_{1}^{m}=y_{1}^{m} ; \text { and }
$$

$\left(c_{2}\right)$ For every $a_{1}^{n} \in Q$ there is at least one $x_{1}^{m} \in Q^{m}$ such that the following equality holds $A\left(a_{1}^{i-1}, x_{1}^{m}, a_{i}^{n-m}\right)=a_{n-m+1}^{n}$. 
2) $\Rightarrow$ : By Def. 1.1.

$3) \Leftarrow$ : Firstly we prove the following statements:

$1^{\prime}(Q ; A)$ is an $(n, m)$-semigroup.

$2^{\prime}$ For every $a_{1}^{n} \in Q$ there is at least one $x_{1}^{m} \in Q^{m}$ such that the following equality holds

$$
A\left(a_{1}^{n-m}, x_{1}^{m}\right)=a_{n-m+1}^{n} .
$$

$3^{\prime}$ For every $a_{1}^{n} \in Q$ there is at least one $y_{1}^{m} \in Q^{m}$ such that the following equality holds

$$
A\left(y_{1}^{m}, a_{1}^{n-m}\right)=a_{n-m+1}^{n} .
$$

The proof of $1^{\prime}$ : By $(a),(b),\left(c_{1}\right)$ and by Prop. $4.2_{3}$.

Sketch of the proof of $2^{\prime}$ :

$$
\begin{aligned}
& A\left(a_{1}^{n-m}, x_{1}^{m}\right)=a_{n-m+1}^{n} \stackrel{\left(c_{1}\right)}{\Longleftrightarrow} 7 \\
& A\left(c_{1}^{i-1}, A\left(a_{1}^{n-m}, x_{1}^{m}\right), c_{i}^{n-m}\right)=A\left(c_{1}^{i-1}, a_{n-m+1}^{n}, c_{i}^{n-m}\right) \stackrel{1^{\prime}}{\Longleftrightarrow} \\
& A\left(c_{1}^{i-1-m}, A\left(c_{i-m}^{i-1}, a_{1}^{n-m}\right), x_{1}^{m}, c_{i}^{n-m}\right)=A\left(c_{1}^{i-1}, a_{n-m+1}^{n}, c_{i}^{n-m}\right),
\end{aligned}
$$

i. e. that

$$
\begin{aligned}
& A\left(a_{1}^{n-m}, x_{1}^{m}\right)=a_{n-m+1}^{n} \Longleftrightarrow \\
& A\left(c_{1}^{i-1-m}, A\left(c_{i-m}^{i-1}, a_{1}^{n-m}\right), x_{1}^{m}, c_{i}^{n-m}\right)=A\left(c_{1}^{i-1}, a_{n-m+1}^{n}, c_{i}^{n-m}\right),
\end{aligned}
$$

where $c_{1}^{n-m}$ is an arbitrary sequence over $Q$.

Whence, by $(c)$, we conclude that the statement $2^{\prime}$ holds.

Remark: Since $n \geq 3 m$ and $i \in\{m+1, \ldots, n-2 m+1\}$, we have $\left|c_{1}^{i-1}\right| \geq m$ and $\left|c_{i}^{n-m}\right| \geq m$.

Similarly, it is possible that the statement $3^{\prime}$ holds.

Finally, by $1^{\prime}-3^{\prime}$ and Th. $5.2_{1}$ (or Th. $5.2_{2}$ ), we conclude that $(Q ; A)$ is an $(n, m)$-group. Whence, by " $\Rightarrow "$, we obtain Th. 5.7.

5.81. Theorem [27]: Let $n \geq 3 m, m \geq 2$ and let $(Q ; A)$ be an $(n, m)$-groupoid. Then, $(Q ; A)$ is an $(n, m)$-group iff there is an mapping $\mathrm{E}$ of the set $Q^{n-2 m}$ into the set $Q^{m}$ such that the laws

(1 $\left.1_{L}\right) A\left(A\left(x_{1}^{n}\right), x_{n+1}^{2 n-m}\right)=A\left(x_{1}, A\left(x_{2}^{n+1}\right), x_{n+2}^{2 n-m}\right)$,

$\left(1_{L m}\right) A\left(A\left(a_{1}^{m}, b_{1}^{n-m}\right), c_{1}^{m}, d_{1}^{n-2 m}\right)=A\left(a_{1}^{m}, A\left(b_{1}^{n-m}, c_{1}^{m}\right), d_{1}^{n-2 m}\right)$,

$\left(\widehat{2_{L}}\right) \quad A\left(a_{1}^{n-2 m}, \mathrm{E}\left(a_{1}^{n-2 m}\right), x_{1}^{m}\right)=x_{1}^{m}$ and

$\left(2_{R}\right) A\left(x_{1}^{m}, a_{1}^{n-2 m}, \mathrm{E}\left(a_{1}^{n-2 m}\right)\right)=x_{1}^{m}$

hold in the algebra $(Q ; A, \mathrm{E})$.

$7 \stackrel{\left(c_{1}\right)}{\Longleftarrow} . \Rightarrow$ : monotony. 
Remark: For $m=1:(1 L m)=(1 L)$. The case $m=1$ is described in [7]. See, also XII-1 in [23].

Proof. 1) $\Rightarrow$ : By Def. 1.1, Th. 3.3 and by Th. 2.10.

$2) \Leftarrow$ : Firstly we prove the following statements:

1 " For every $x_{1}^{m}, y_{1}^{m}, b_{1}^{m} \in Q^{m}$ and for every sequence $a_{1}^{n-2 m}$ over $Q$ the following implication holds

$A\left(x_{1}^{m}, b_{1}^{m}, a_{1}^{n-2 m}\right)=A\left(y_{1}^{m}, b_{1}^{m}, a_{1}^{n-2 m}\right) \Rightarrow x_{1}^{m}=y_{1}^{m}$.

$2 "(Q ; A)$ is an $(n, m)$-semigroup.

3" $\left(\forall b_{1}^{m} \in Q^{m}\right)\left(\forall c_{i} \in Q\right)_{1}^{n-3 m} b_{1}^{m}=\mathrm{E}\left(c_{1}^{n-3 m}, \mathrm{E}\left(b_{1}^{m}, c_{1}^{n-3 m}\right)\right)$.

4" For every $x_{1}^{m}, y_{1}^{m}, b_{1}^{m} \in Q^{m}$ and for every sequence $a_{1}^{n-2 m}$ over $Q$ the following implication

$$
A\left(b_{1}^{m}, x_{1}^{m}, a_{1}^{n-2 m}\right)=A\left(b_{1}^{m}, y_{1}^{m}, a_{1}^{n-2 m}\right) \Rightarrow x_{1}^{m}=y_{1}^{m}
$$

holds.

5" For every $x_{1}^{m}, y_{1}^{m}, b_{1}^{m} \in Q^{m}$ and for every sequence $a_{1}^{n-2 m}$ over $Q$ the following implication

$$
A\left(a_{1}^{n-2 m}, x_{1}^{m}, b_{1}^{m}\right)=A\left(a_{1}^{n-2 m}, y_{1}^{m}, b_{1}^{m}\right) \Rightarrow x_{1}^{m}=y_{1}^{m}
$$

holds.

6" For every $x_{1}^{m}, b_{1}^{m}, d_{1}^{m} \in Q^{m}$ and for every sequence $a_{1}^{n-2 m}$ over $Q$ the following equivalence holds

$$
\begin{aligned}
& \quad A\left(b_{1}^{m}, x_{1}^{m}, a_{1}^{n-2 m}\right)=d_{1}^{m} \Leftrightarrow \\
& x_{1}^{m}=A\left(c_{1}^{n-3 m}, \mathrm{E}\left(b_{1}^{m}, c_{1}^{n-3 m}\right), d_{1}^{m}, \mathrm{E}\left(a_{1}^{n-2 m}\right)\right), \\
& \text { where } c_{1}^{n-3 m} \text { arbitrary sequence over } Q .
\end{aligned}
$$

Sketch of the proof of 1":

$$
\begin{aligned}
& A\left(x_{1}^{m}, b_{1}^{m}, a_{1}^{n-2 m}\right)=A\left(y_{1}^{m}, b_{1}^{m}, a_{1}^{n-2 m}\right) \stackrel{n \geq 3 m}{\Longrightarrow} \\
& A\left(A\left(x_{1}^{m}, b_{1}^{m}, a_{1}^{n-2 m}\right), \mathrm{E}\left(a_{1}^{n-2 m}\right), c_{1}^{n-3 m}, \mathrm{E}\left(b_{1}^{m}, c_{1}^{n-3 m}\right)\right)= \\
& A\left(A\left(y_{1}^{m}, b_{1}^{m}, a_{1}^{n-2 m}\right), \mathrm{E}\left(a_{1}^{n-2 m}\right), c_{1}^{n-3 m}, \mathrm{E}\left(b_{1}^{m}, c_{1}^{n-3 m}\right)\right) \stackrel{(1 L m)}{\Longrightarrow} \\
& A\left(x_{1}^{m}, A\left(b_{1}^{m}, a_{1}^{n-2 m}, \mathrm{E}\left(a_{1}^{n-2 m}\right)\right), c_{1}^{n-3 m}, \mathrm{E}\left(b_{1}^{m}, c_{1}^{n-3 m}\right)\right)= \\
& A\left(y_{1}^{m}, A\left(b_{1}^{m}, a_{1}^{n-2 m}, \mathrm{E}\left(a_{1}^{n-2 m}\right)\right), c_{1}^{n-3 m}, \mathrm{E}\left(b_{1}^{m}, c_{1}^{n-3 m}\right)\right) \stackrel{(2 R)}{\Longrightarrow} \\
& A\left(x_{1}^{m}, b_{1}^{m}, c_{1}^{n-3 m}, \mathrm{E}\left(b_{1}^{m}, c_{1}^{n-3 m}\right)\right)=(2 R) \\
& A\left(y_{1}^{m}, b_{1}^{m}, c_{1}^{n-3 m}, \mathrm{E}\left(b_{1}^{m}, c_{1}^{n-3 m}\right)\right) \stackrel{(2)}{\Longrightarrow} x_{1}^{m}=y_{1}^{m} .
\end{aligned}
$$

The proof of 2" : By 1", $(1 L)$ and by Prop. 4.2.

Sketch of the proof of 3" :

$$
A\left(b_{1}^{m}, c_{1}^{n-3 m}, \mathrm{E}\left(b_{1}^{m}, c_{1}^{n-3 m}\right), \mathrm{E}\left(c_{1}^{n-3 m}, \mathrm{E}\left(b_{1}^{m}, c_{1}^{n-3 m}\right)\right) \stackrel{(\widehat{2 L})}{=}\right.
$$




$$
\begin{aligned}
& \mathrm{E}\left(c_{1}^{n-3 m}, \mathrm{E}\left(b_{1}^{m}, c_{1}^{n-3 m}\right)\right) \\
& A\left(b_{1}^{m}, c_{1}^{n-3 m}, \mathrm{E}\left(b_{1}^{m}, c_{1}^{n-3 m}\right), \mathrm{E}\left(c_{1}^{n-3 m}, \mathrm{E}\left(b_{1}^{m}, c_{1}^{n-3 m}\right)\right)\right) \stackrel{(2 R)}{=} b_{1}^{m} \text {. }
\end{aligned}
$$

Sketch of the proof of $5 "$ :

$A\left(a_{1}^{n-2 m}, x_{1}^{m}, b_{1}^{m}\right)=A\left(a_{1}^{n-2 m}, y_{1}^{m}, b_{1}^{m}\right) \stackrel{n \geq 3 m}{\Longrightarrow}$

$A\left(c_{1}^{2 m}, A\left(a_{1}^{n-2 m}, x_{1}^{m}, b_{1}^{m}\right), d_{1}^{n-3 m}\right)=$ $A\left(c_{1}^{2 m}, A\left(a_{1}^{n-2 m}, y_{1}^{m}, b_{1}^{m}\right), d_{1}^{n-3 m}\right) \stackrel{2 ”}{\Longrightarrow}$ $A\left(A\left(c_{1}^{2 m}, a_{1}^{n-2 m}\right), x_{1}^{m}, b_{1}^{m}, d_{1}^{n-3 m}\right)=$ $A\left(A\left(c_{1}^{2 m}, a_{1}^{n-2 m}\right), y_{1}^{m}, b_{1}^{m}, d_{1}^{n-3 m}\right) \stackrel{4^{\prime \prime}}{\Longrightarrow} x_{1}^{m}=y_{1}^{m}$.

Sketch of the proof of 6 " :

$$
\begin{aligned}
& A\left(b_{1}^{m}, x_{1}^{m}, a_{1}^{n-2 m}\right)=d_{1}^{m} \stackrel{5^{\prime \prime}}{\Longleftrightarrow} 8 \\
& A\left(c_{1}^{n-3 m}, \mathrm{E}\left(b_{1}^{m}, c_{1}^{n-3 m}\right), A\left(b_{1}^{m}, x_{1}^{m}, a_{1}^{n-2 m}\right), \mathrm{E}\left(a_{1}^{n-2 m}\right)\right)= \\
& A\left(c_{1}^{n-3 m}, \mathrm{E}\left(b_{1}^{m}, c_{1}^{n-3 m}\right), d_{1}^{m}, \mathrm{E}\left(a_{1}^{n-2 m}\right)\right) \stackrel{2^{\prime \prime}}{\Longleftrightarrow} \\
& A\left(c_{1}^{n-3 m}, \mathrm{E}\left(b_{1}^{m}, c_{1}^{n-3 m}\right), b_{1}^{m}, A\left(x_{1}^{m}, a_{1}^{n-2 m}, \mathrm{E}\left(a_{1}^{n-2 m}\right)\right)\right)= \\
& A\left(c_{1}^{n-3 m}, \mathrm{E}\left(b_{1}^{m}, c_{1}^{n-3 m}\right), d_{1}^{m}, \mathrm{E}\left(a_{1}^{n-2 m}\right)\right) \stackrel{(2 R)}{\Longleftrightarrow} \\
& A\left(c_{1}^{n-3 m}, \mathrm{E}\left(b_{1}^{m}, c_{1}^{n-3 m}\right), b_{1}^{m}, x_{1}^{m}\right)= \\
& A\left(c_{1}^{n-3 m}, \mathrm{E}\left(b_{1}^{m}, c_{1}^{n-3 m}\right), d_{1}^{m}, \mathrm{E}\left(a_{1}^{n-2 m}\right)\right) \stackrel{3^{\prime \prime}}{\Longleftrightarrow} \\
& A\left(c_{1}^{n-3 m}, \mathrm{E}\left(b_{1}^{m}, c_{1}^{n-3 m}\right), \mathrm{E}\left(c_{1}^{n-3 m}, \mathrm{E}\left(b_{1}^{m}, c_{1}^{n-3 m}\right)\right), x_{1}^{m}\right)= \\
& A\left(c_{1}^{n-3 m}, \mathrm{E}\left(b_{1}^{m}, c_{1}^{n-3 m}\right), d_{1}^{m}, \mathrm{E}\left(a_{1}^{n-2 m}\right)\right) \stackrel{(\widehat{2 L})}{\Longleftrightarrow} \\
& x_{1}^{m}=A\left(c_{1}^{n-3 m}, \mathrm{E}\left(b_{1}^{m}, c_{1}^{n-3 m}\right), d_{1}^{m}, \mathrm{E}\left(a_{1}^{n-2 m}\right)\right) .
\end{aligned}
$$

Finally, by $2 ", 4 ", 6 "$ and by Th. 5.7 , we conclude that $(Q ; A)$ is an $(n, m)$-group. Whence, by " $\Rightarrow "$, we obtain Th. $5.8_{1}$

$8 \stackrel{5 "}{\Longleftarrow} . \Rightarrow:$ monotony. 
Similarly, one could prove also the following proposition:

5.82. Theorem [27]: Let $n \geq 3 m, m \geq 2$ and let $(Q ; A)$ be an $(n, m)$-groupoid. Then, $(Q ; A)$ is an $(n, m)$-group iff there is an mapping $\mathrm{E}$ of the set $Q^{n-2 m}$ into the set $Q^{m}$ such that the laws

(1 $R) \quad A\left(x_{1}^{n-m-1}, A\left(x_{n-m}^{2 n-m-1}\right), x_{2 n-m}\right)=A\left(x_{1}^{n-m}, A\left(x_{n-m+1}^{2 n-m}\right)\right)$,

$\left(1_{R m}\right) A\left(a_{1}^{n-2 m}, A\left(b_{1}^{m}, c_{1}^{n-m}\right), d_{1}^{m}\right)=A\left(a_{1}^{n-2 m}, b_{1}^{m}, A\left(c_{1}^{n-m}, d_{1}^{m}\right)\right)$,

$\left(2_{L}\right) \quad A\left(\mathrm{E}\left(a_{1}^{n-2 m}\right), a_{1}^{n-2 m}, x_{1}^{m}\right)=x_{1}^{m}$ and

$\left(\widehat{2_{R}}\right) \quad A\left(x_{1}^{m}, \mathrm{E}\left(a_{1}^{n-2 m}\right), a_{1}^{n-2 m}\right)=x_{1}^{m}$

hold in the algebra $(Q ; A, \mathrm{E})$.

Remark: For $m=1:(1 R m)=(1 R)$. The case $m=1$ is described in [7]. See, also XII-1 in [23].

\section{About $(k m, m)$-GROUPS FOR $k>2$ AND $m \geq 2$}

6.1. Theorem [24]: Let $k>2, m \geq 2, n=k \cdot m,(Q ; A)(n, m)$-group and $\mathbf{e}$ its $\{1, n-m+1\}$-neutral operation. Also let there exist a sequence $a_{1}^{n-2 m}$ over $Q$ such that for all $i \in\{0,1, \ldots, 2 m-1\}$, and for every $x_{1}^{2 m} \in Q$ the following equality holds

(0) $A\left(x_{1}^{i}, a_{1}^{n-2 m}, x_{i+1}^{2 m}\right)=A\left(x_{1}^{2 m}, a_{1}^{n-2 m}\right)$.

Further on, let

(1) $B\left(x_{1}^{2 m}\right) \stackrel{\text { def }}{=} A\left(x_{1}^{m}, a_{1}^{n-2 m}, x_{m+1}^{2 m}\right)$ and

(2) $c_{1}^{m} \stackrel{\text { def }}{=} A\left(\frac{k}{\mathbf{e}\left(a_{1}^{n-2 m}\right)}\right)$

for all $x_{1}^{2 m} \in Q$. Then the following statements hold

(i) $(Q ; B)$ is a $(2 m, m)$-group;

(ii) For all $x_{1}^{k \cdot m} \in Q$

$$
A\left(x_{1}^{k \cdot m}\right)=\stackrel{k}{B}\left(x_{1}^{k \cdot m}, c_{1}^{m}\right) ; \text { and }
$$

(iii) For all $j \in\{0, \ldots, m-1\}$ and for every $x_{1}^{m} \in Q$ the following equality holds

$$
B\left(x_{1}^{j}, c_{1}^{m}, x_{j+1}^{m}\right)=B\left(x_{1}^{m}, c_{1}^{m}\right) .
$$

Proof. Firstly we prove the following statements:

$1^{\circ}$ For all $x_{1}^{3 m} \in Q$ the following equality holds

$$
B\left(B\left(x_{1}^{2 m}\right), x_{2 m+1}^{3 m}\right)=B\left(x_{1}, B\left(x_{2}^{2 m+1}\right), x_{2 m+2}^{3 m}\right) .
$$


$2^{\circ}$ For all $b_{1}^{2 m} \in Q$ there is exactly one $x_{1}^{m} \in Q^{m}$ such that the following equality holds

$$
B\left(x_{1}^{m}, b_{1}^{m}\right)=b_{m+1}^{2 m} .
$$

$3^{\circ}(Q ; B)$ is a $(2 m, m)$-semigroup.

$4^{\circ}$ For all $b_{1}^{2 m} \in Q$ there is exactly one $y_{1}^{m} \in Q^{m}$ such that the following equality holds

$$
B\left(b_{1}^{m}, y_{1}^{m}\right)=b_{m+1}^{2 m} .
$$

Sketch of the proof of $1^{\circ}$ :

$B\left(B\left(x_{1}^{2 m}\right), x_{2 m+1}^{3 m}\right) \stackrel{(1)}{=}$

$A\left(A\left(x_{1}^{m}, a_{1}^{n-2 m}, x_{m+1}^{2 m}\right), a_{1}^{n-2 m}, x_{2 m+1}^{3 m}\right) \stackrel{(0)}{=}$

$A\left(A\left(x_{1}^{m}, a_{1}^{n-2 m}, x_{m+1}^{2 m}\right), x_{2 m+1}, a_{1}^{n-2 m}, x_{2 m+2}^{3 m}\right) \stackrel{1.1(I)}{=}$

$A\left(x_{1}, A\left(x_{2}^{m}, a_{1}^{n-2 m}, x_{m+1}^{2 m}, x_{2 m+1}\right), a_{1}^{n-2 m}, x_{2 m+2}^{3 m}\right) \stackrel{(0)(1)}{=}$

$B\left(x_{1}, B\left(x_{2}^{2 m+1}\right), x_{2 m+2}^{3 m}\right)$.

Sketch of the proof of $2^{\circ}$ :

$B\left(x_{1}^{m}, b_{1}^{m}\right)=b_{m+1}^{2 m} \stackrel{(1)}{\Leftrightarrow}$

$A\left(x_{1}^{m}, a_{1}^{n-2 m}, b_{1}^{m}\right)=b_{m+1}^{2 m}$,

whence, by Def. 1.1-(II), we obtain $2^{\circ}$.

Sketch of the proof of $3^{\circ}$ :

By $2^{\circ}$ and by Prop. 2.1.

Sketch of the proof of $4^{\circ}$ :

$B\left(b_{1}^{m}, x_{1}^{m}\right)=b_{m+1}^{2 m} \stackrel{(1)}{\Leftrightarrow}$

$A\left(b_{1}^{m}, a_{1}^{n-2 m}, y_{1}^{m}\right)=b_{m+1}^{2 m}$,

whence, by Def. 1.1-(II), we have $4^{\circ}$.

The proof of $(i)$ :

By $2^{\circ}, 3^{\circ}, 4^{\circ}$ and by Prop.2.2.

Sketch of the proof of ( $i i)$ [to the case $k=4$ ]:

$$
\begin{aligned}
& A\left(x_{1}^{m}, y_{1}^{m}, z_{1}^{m}, u_{1}^{m}\right) \stackrel{2.5}{=} \\
& A\left(x_{1}^{m}, y_{1}^{m}, z_{1}^{m}, A\left(u_{1}^{m}, a_{1}^{n-2 m}, \mathbf{e}\left(a_{1}^{n-2 m}\right)\right)\right) \stackrel{1.1(I)}{=} \\
& A\left(x_{1}^{m}, y_{1}^{m}, A\left(z_{1}^{m}, u_{1}^{m}, a_{1}^{n-2 m}\right), \mathbf{e}\left(a_{1}^{n-2 m}\right)\right) \stackrel{(0)}{=} \\
& A\left(x_{1}^{m}, y_{1}^{m}, A\left(z_{1}^{m}, a_{1}^{n-2 m}, u_{1}^{m}\right), \mathbf{e}\left(a_{1}^{n-2 m}\right)\right) \stackrel{(1)}{=} \\
& A\left(x_{1}^{m}, y_{1}^{m}, B\left(z_{1}^{m}, u_{1}^{m}\right), \mathbf{e}\left(a_{1}^{n-2 m}\right)\right) \stackrel{2.5}{=} \\
& A\left(x_{1}^{m}, y_{1}^{m}, A\left(B\left(z_{1}^{m}, u_{1}^{m}\right), a_{1}^{n-2 m}, \mathbf{e}\left(a_{1}^{n-2 m}\right)\right), \mathbf{e}\left(a_{1}^{n-2 m}\right)\right) \stackrel{1.1(I)}{=}
\end{aligned}
$$




$$
\begin{aligned}
& A\left(x_{1}^{m}, A\left(y_{1}^{m}, B\left(z_{1}^{m}, u_{1}^{m}\right), a_{1}^{n-2 m}\right), \mathbf{e}\left(a_{1}^{n-2 m}\right), \mathbf{e}\left(a_{1}^{n-2 m}\right)\right) \stackrel{(0)}{=} \\
& A\left(x_{1}^{m}, A\left(y_{1}^{m}, a_{1}^{n-2 m}, B\left(z_{1}^{m}, u_{1}^{m}\right)\right), \mathbf{e}\left(a_{1}^{n-2 m}\right), \mathbf{e}\left(a_{1}^{n-2 m}\right)\right) \stackrel{(1)}{=} \\
& A\left(x_{1}^{m}, B\left(y_{1}^{m}, B\left(z_{1}^{m}, u_{1}^{m}\right)\right), \mathbf{e}\left(a_{1}^{n-2 m}\right), \mathbf{e}\left(a_{1}^{n-2 m}\right)\right) \stackrel{2.5}{=} \\
& A\left(x_{1}^{m}, A\left(B\left(y_{1}^{m}, B\left(z_{1}^{m}, u_{1}^{m}\right)\right), a_{1}^{n-2 m}, \mathbf{e}\left(a_{1}^{n-2 m}\right)\right), \mathbf{e}\left(a_{1}^{n-2 m}\right), \mathbf{e}\left(a_{1}^{n-2 m}\right)\right) \stackrel{1.1(I)}{=} \\
& A\left(A\left(x_{1}^{m}, B\left(y_{1}^{m}, B\left(z_{1}^{m}, u_{1}^{m}\right)\right), a_{1}^{n-2 m}\right), \mathbf{e}\left(a_{1}^{n-2 m}\right), \mathbf{e}\left(a_{1}^{n-2 m}\right), \mathbf{e}\left(a_{1}^{n-2 m}\right)\right) \stackrel{(0)}{=} \\
& A\left(A\left(x_{1}^{m}, a_{1}^{n-2 m}, B\left(y_{1}^{m}, B\left(z_{1}^{m}, u_{1}^{m}\right)\right)\right), \mathbf{e}\left(a_{1}^{n-2 m}\right), \mathbf{e}\left(a_{1}^{n-2 m}\right), \mathbf{e}\left(a_{1}^{n-2 m}\right)\right) \stackrel{(1)}{=} \\
& A\left(B\left(x_{1}^{m}, B\left(y_{1}^{m}, B\left(z_{1}^{m}, u_{1}^{m}\right)\right), \mathbf{e}\left(a_{1}^{n-2 m}\right),\left(\overline{\mathbf{e}\left(a_{1}^{n-2 m}\right)}\right)\right) \stackrel{4.4}{=}\right. \\
& A\left(\stackrel{3}{B}\left(x_{1}^{m}, y_{1}^{m}, z_{1}^{m}, u_{1}^{m}\right)\right), A\left(\mathbf{e}\left(a_{1}^{n-2}\right), a_{1}^{n-2}, \mathbf{e}\left(a_{1}^{n-2 m}\right)\right),\left(\overline{\mathbf{e}\left(a_{1}^{n-2 m}\right)}\right) \stackrel{(0)}{=} \\
& \left.A\left(\stackrel{3}{B}\left(x_{1}^{m}, y_{1}^{m}, z_{1}^{m}, u_{1}^{m}\right), A\left(a_{1}^{n-2 m}, \mathbf{e}\left(a_{1}^{n-2 m}\right), \mathbf{e}\left(a_{1}^{n-2 m}\right)\right), \overline{\mathbf{e}\left(a_{1}^{n-2 m}\right)}\right)\right) \stackrel{1.1(I)}{=} \\
& A\left(\stackrel{3}{B}\left(x_{1}^{m}, y_{1}^{m}, z_{1}^{m}, u_{1}^{m}\right), a_{1}^{n-2 m}, A\left(\overline{\mathbf{e}\left(a_{1}^{n-2 m}\right)} \mid\right)\right) \stackrel{(1)}{=} \\
& B\left(\stackrel{3}{B}\left(x_{1}^{m}, y_{1}^{m}, z_{1}^{m}, u_{1}^{m}\right), A\left(\overline{\mathbf{e}\left(a_{1}^{n-2 m}\right)} \mid\right)\right) \stackrel{(2)}{=} \\
& B\left(\stackrel{3}{B}\left(x_{1}^{m}, y_{1}^{m}, z_{1}^{m}, u_{1}^{m}\right), c_{1}^{m}\right) \stackrel{4.3}{=} \\
& \stackrel{4}{B}\left(x_{1}^{m}, y_{1}^{m}, z_{1}^{m}, u_{1}^{m}, c_{1}^{m}\right) \text {. }
\end{aligned}
$$

Sketch of a part of the proof of $($ iii) :

By $(i i)$ and by

$$
A\left(A\left(x_{1}^{k \cdot m}\right), x_{k \cdot m+1}^{2 k m-m}\right)=A\left(x_{1}, A\left(x_{2}^{k \cdot m+1}\right), x_{k \cdot m+2}^{2 k m-m}\right),
$$

we have

$\stackrel{k}{B}\left(x_{1}, \stackrel{k}{B}\left(x_{2}^{k \cdot m}, c_{1}^{m}, x_{k \cdot m+1}\right), x_{k \cdot m+2}^{2 k m-m}, c_{1}^{m}\right)=$

$\stackrel{k}{B}\left(x_{1}, \stackrel{k}{B}\left(x_{2}^{k \cdot m}, c_{1}^{m}\right), x_{k \cdot m+2}^{2 k m-m}, c_{1}^{m}\right)$,

and by Def.1.1-(II), we have

$\stackrel{k}{B}\left(x_{2}^{k \cdot m}, c_{1}^{m}, x_{k \cdot m+1}\right)=\stackrel{k}{B}\left(x_{2}^{k \cdot m+1}, c_{1}^{m}\right)$,

i.e., by Prop. 4.4,

$$
\begin{aligned}
& { }_{B}^{k-1}\left(x_{2}^{(k-1) \cdot m+1}, B\left(x_{(k-1) \cdot m+2}^{k \cdot m}, c_{1}^{m}, x_{k \cdot m+1}\right)\right)= \\
& k-1\left(x_{2}^{(k-1) \cdot m+1}, B\left(x_{(k-1) \cdot m+2}^{k \cdot m+1}, c_{1}^{m}\right)\right) .
\end{aligned}
$$

Finaly, hence we obtain

$$
B\left(x_{(k-1) \cdot m+2}^{k \cdot m}, c_{1}^{m}, x_{k \cdot m+1}\right)=B\left(x_{(k-1) \cdot m+2}^{k \cdot m+1}, c_{1}^{m}\right),
$$

i.e., we obtain $(i i i)$ for $j=m-1$. 
6.2. Theorem [24]: Let $m \geq 2,(Q ; B)$ be a $(2 m, m)$-group, and let $\stackrel{m}{e} \in Q^{m}$ its neutral element (cf. Prop. 2.9). Also let $c_{1}^{m}$ be an element of the set $Q^{m}$ such that for every $i \in\{0,1, \ldots, m-1\}$ and for every $x_{1}^{m} \in Q^{m}$ the following equality holds

(a) $B\left(x_{1}^{i}, c_{1}^{m}, x_{i+1}^{m}\right)=B\left(x_{1}^{m}, c_{1}^{m}\right)$

(cf. Prop. 2.6 and Prop. 2.7). Further on, let $k>2$ and

(b) $A\left(x_{1}^{k \cdot m}\right)=\stackrel{k}{B}\left(x_{1}^{k \cdot m}, c_{1}^{m}\right)$

for all $x_{1}^{k \cdot m} \in Q$. Then $(Q ; A)$ is a $(k m, m)$-group with condition:

(c) There exists a sequence $a_{1}^{(k-2) \cdot m}$ over $Q$ such that for all $j \in\{0, \ldots, 2 m-1\}$ and for every $x_{1}^{2 m} \in Q$ the following equality holds

$A\left(x_{1}^{j}, a_{1}^{(k-2) \cdot m}, x_{j+1}^{2 m}\right)=A\left(x_{1}^{2 m}, a_{1}^{(k-2) \cdot m}\right)$.

Proof. Firstly we prove the following statements:

$\stackrel{\circ}{1}$ For all $x_{1}^{2 k m-m} \in Q$ the following equality holds

$A\left(A\left(x_{1}^{k \cdot m}\right), x_{k \cdot m+1}^{2 k m-m}\right)=A\left(x_{1}, A\left(x_{2}^{k \cdot m+1}\right), x_{k \cdot m+2}^{2 k m-m}\right)$

$[<1,2>-$ associative law $]$.

$\stackrel{\circ}{2}$ For all $b_{1}^{2 k m} \in Q$ there is exactly one $x_{1}^{m} \in Q^{m}$ such that the following equality holds

$A\left(x_{1}^{m}, b_{1}^{k \cdot m-m}\right)=b_{k \cdot m-m+1}^{k \cdot m}$.

$\stackrel{\circ}{3}(Q ; A)$ is a $(k m, m)$-semigroup.

$\stackrel{\circ}{4}$ For all $b_{1}^{2 k m} \in Q$ there is exactly one $y_{1}^{m} \in Q^{m}$ such that the following equality holds

$$
A\left(b_{1}^{k \cdot m-m}, y_{1}^{m}\right)=b_{k \cdot m-m+1}^{k \cdot m} .
$$

$\stackrel{\circ}{5}$ For all $j \in\{0, \ldots, 2 m-1\}$ and for every $x_{1}^{2 k m} \in Q$ the following equality holds

$$
A\left(x_{1}^{j}, \stackrel{(k-3) \cdot m}{e},\left(c_{1}^{m}\right)^{-1}, x_{j+1}^{2 m}\right)=A\left(x_{1}^{2 m}, \stackrel{(k-3) \cdot m}{e},\left(c_{1}^{m}\right)^{-1}\right)
$$

where

(d) $B\left(\left(c_{1}^{m}\right)^{-1}, c_{1}^{m}\right)=\stackrel{m}{e}$ [cf. Prop. 1.5 and Prop. 2.8].

Sketch of the proof of $\stackrel{\circ}{1}$ :

$$
\begin{aligned}
& A\left(A\left(x_{1}^{k \cdot m}\right), x_{k \cdot m+1}^{2 k m-m}\right) \stackrel{(b)}{=} \\
& \stackrel{k}{B}\left(\stackrel{k}{B}\left(x_{1}^{k \cdot m}, c_{1}^{m}\right), x_{k \cdot m+1}^{2 k m-m}, c_{1}^{m}\right) \stackrel{4.5}{=} \\
& \stackrel{k}{B}\left(x_{1}, \stackrel{k}{B}\left(x_{2}^{k \cdot m}, c_{1}^{m}, x_{k \cdot m+1}\right), x_{k \cdot m+2}^{2 k m-m}, c_{1}^{m}\right) \stackrel{4.4}{=}
\end{aligned}
$$


$\stackrel{k}{B}\left(x_{1}, \stackrel{k-1}{B}\left(x_{2}^{(k-1) \cdot m+1}, B\left(x_{(k-1) \cdot m+2}^{k \cdot m}, c_{1}^{m}, x_{k \cdot m+1}\right)\right), x_{k \cdot m+2}^{2 k m-m}, c_{1}^{m}\right) \stackrel{(a)}{=}$

$\stackrel{k}{B}\left(x_{1}, \stackrel{k-1}{B}\left(x_{2}^{(k-1) \cdot m+1}, B\left(x_{(k-1) \cdot m+2}^{k \cdot m+1}, c_{1}^{m}\right)\right), x_{k \cdot m+2}^{2 k m-m}, c_{1}^{m}\right) \stackrel{4.4}{=}$

$\stackrel{k}{B}\left(x_{1}, \stackrel{k}{B}\left(x_{2}^{k \cdot m+1}, c_{1}^{m}\right), x_{k \cdot m+2}^{2 k m-m}, c_{1}^{m}\right) \stackrel{(b)}{=}$

$A\left(x_{1}, A\left(x_{2}^{k \cdot m+1}\right), x_{k \cdot m+2}^{2 k m-m}\right)$.

Sketch of the proof of $\stackrel{\circ}{2}$ :

$A\left(x_{1}^{m}, b_{1}^{(k-1) \cdot m}\right)=b_{(k-1) \cdot m+1}^{k \cdot m} \stackrel{(b)}{\Leftrightarrow}$

$\stackrel{k}{B}\left(x_{1}^{m}, b_{1}^{(k-1) \cdot m}, c_{1}^{m}\right)=b_{(k-1) \cdot m+1}^{k \cdot m} \stackrel{4.5}{\Leftrightarrow}$

$B\left(x_{1}^{m},\left(\stackrel{k-1}{B} b_{1}^{(k-1) \cdot m}, c_{1}^{m}\right)\right)=b_{(k-1) \cdot m+1}^{k \cdot m}$.

Sketch of the proof of 3 :

By $\stackrel{\circ}{1}, \stackrel{\circ}{2}$ and by Prop. 2.1.

Sketch of the proof of 4 :

$A\left(b_{1}^{(k-1) \cdot m}, y_{1}^{m}\right)=b_{(k-1) \cdot m+1}^{k \cdot m} \stackrel{(b)}{\Leftrightarrow}$

$\stackrel{k}{B}\left(b_{1}^{(k-1) \cdot m}, y_{1}^{m}, c_{1}^{m}\right)=b_{(k-1) \cdot m+1}^{k \cdot m} \stackrel{4.4}{\Leftrightarrow}$

$\stackrel{k-1}{B}\left(b_{1}^{(k-1) \cdot m}, B\left(y_{1}^{m}, c_{1}^{m}\right)\right)=b_{(k-1) \cdot m+1}^{k \cdot m} \stackrel{(a) i=0}{\Longleftrightarrow}$

$\stackrel{k-1}{B}\left(b_{1}^{(k-1) \cdot m}, B\left(c_{1}^{m}, y_{1}^{m}\right)\right)=b_{(k-1) \cdot m+1}^{k \cdot m} \stackrel{4.4}{\Longleftrightarrow}$

$\stackrel{k}{B}\left(b_{1}^{(k-1) \cdot m}, c_{1}^{m}, y_{1}^{m}\right)=b_{(k-1) \cdot m+1}^{k \cdot m} \stackrel{4.3}{\Longleftrightarrow}$

$B\left(\stackrel{k-1}{B}\left(b_{1}^{(k-1) \cdot m}, c_{1}^{m}\right), y_{1}^{m}\right)=b_{(k-1) \cdot m+1}^{k \cdot m}$.

Sketch of a part of the proof of $\stackrel{\circ}{5}$ [to case $k=4$ ]:

$A\left(x_{1}^{2 m}, \stackrel{m}{e},\left(c_{1}^{m}\right)^{-1}\right) \stackrel{(b)}{=}$

$\stackrel{4}{B}\left(x_{1}^{2 m}, \stackrel{m}{e},\left(c_{1}^{m}\right)^{-1}, c_{1}^{m}\right) \stackrel{4.4}{=}$

$\stackrel{3}{B}\left(x_{1}^{2 m}, \stackrel{m}{e}, B\left(\left(c_{1}^{m}\right)^{-1}, c_{1}^{m}\right)\right) \stackrel{(d)}{=}$

$\stackrel{3}{B}\left(x_{1}^{2 m}, \stackrel{m}{e}, \stackrel{m}{e}\right) \stackrel{4.4}{=}$

$\stackrel{2}{B}\left(x_{1}^{m}, B\left(x_{m+1}^{2 m}, \stackrel{m}{e}\right), \stackrel{m}{e}\right) \stackrel{(a)}{=}$

$\stackrel{2}{B}\left(x_{1}^{m}, B\left(x_{m+1}^{2 m-1}, \stackrel{m}{e}, x_{2 m}\right), \stackrel{m}{e}\right) \stackrel{4.4}{=}$

$\stackrel{2}{B}\left(x_{1}^{2 m-1}, e, B\left(\stackrel{m-1}{e}, x_{2 m}, \stackrel{m}{e}\right)\right) \stackrel{(a)}{=}$

$\stackrel{2}{B}\left(x_{1}^{2 m-1}, e, B\left(\stackrel{m-1}{e}, \stackrel{m}{e}, x_{2 m}\right)\right) \stackrel{4.4}{=}$

$\stackrel{3}{B}\left(x_{1}^{2 m-1}, \stackrel{m}{e}, \stackrel{m}{e}, x_{2 m}\right) \stackrel{(d)}{=}$ 


$$
\begin{aligned}
& \stackrel{3}{B}\left(x_{1}^{2 m-1}, \stackrel{m}{e}, B\left(\left(c_{1}^{m}\right)^{-1}, c_{1}^{m}\right), x_{2 m}\right)=9 \\
& \stackrel{3}{B}\left(x_{1}^{2 m-1}, \stackrel{m}{e}, B\left(\left(\bar{c}_{1}^{m}, c_{1}^{m}\right), x_{2 m}\right) \stackrel{4.4}{=}\right. \\
& \stackrel{3}{B}\left(x_{1}^{2 m-1}, \stackrel{m}{e}, \bar{c}_{1}, B\left(\left(\bar{c}_{2}^{m}, c_{1}^{m}, x_{2 m}\right)\right) \stackrel{(a)}{=}\right. \\
& \stackrel{3}{B}\left(x_{1}^{2 m-1}, \stackrel{m}{e}, \bar{c}_{1}, B\left(\bar{c}_{2}^{m}, x_{2 m}, c_{1}^{m}\right)\right) \stackrel{4.4}{=} \\
& \stackrel{4}{B}\left(x_{1}^{2 m-1}, \stackrel{m}{e}, \bar{c}_{1}^{m}, \bar{c}_{2}^{m}, x_{2 m}, c_{1}^{m}\right)= \\
& \stackrel{4}{B}\left(x_{1}^{2 m-1}, \stackrel{m}{e},\left(c_{1}^{m}\right)^{-1}, x_{2 m}, c_{1}^{m}\right) \stackrel{(b)}{=} \\
& A\left(x_{1}^{2 m-1}, \stackrel{m}{e},\left(c_{1}^{m}\right)^{-1}, x_{2 m}\right) .
\end{aligned}
$$

By $\stackrel{\circ}{2}-\stackrel{\circ}{4}$, Prop. 4.1 and by $\stackrel{\circ}{5}$, we obtain $(Q ; A)$ is a $(k m, m)$-group with condition $(c)$.

6.3. Remarks: a) In [3] the following proposition is proved. Let $(Q ; A)$ be a (km, $m)$-group, $m \geq 2, k \geq 3$ and let

$$
\mathbf{A}\left(x_{1}^{m}, x_{m+1}^{2 m}, \ldots, x_{(k-1) \cdot m+1}^{k \cdot m}\right) \stackrel{\text { def }}{=} A\left(x_{1}^{k \cdot m}\right)
$$

for all $x_{1}^{k \cdot m} \in Q$. Then there exist binary group $\left(Q^{m}, \mathbf{B}\right)$, an element $c_{1}^{m} \in Q^{m}$ and an automorphism $\varphi$ of this group, such that for each $x_{1}^{m}, x_{m+1}^{2 m}, \ldots, x_{(k-1) \cdot m+1}^{k \cdot m} \in$ $Q^{m}$

$$
\begin{aligned}
& \mathbf{A}\left(x_{1}^{m}, x_{m+1}^{2 m}, \ldots, x_{(k-1) \cdot m+1}^{k \cdot m}\right)= \\
& \stackrel{k}{\mathbf{B}}\left(x_{1}^{m}, \varphi\left(x_{m+1}^{2 m}\right), \ldots, \varphi^{k-1}\left(x_{(k-1) \cdot m+1}^{k \cdot m}\right), c_{1}^{m}\right) \text {, } \\
& \varphi\left(c_{1}^{m}\right)=c_{1}^{m} \text { and } \\
& \mathbf{B}\left(\varphi^{k-1}\left(x_{1}^{m}\right), c_{1}^{m}\right)=\mathbf{B}\left(c_{1}^{m}, x_{1}^{m}\right) .
\end{aligned}
$$

b) $\mathbf{B}, \varphi$ and $c_{1}^{m}$ from a), according to [16], are $\underset{k}{\text { defined }}$ in the following way

$$
\begin{aligned}
& \mathbf{B}\left(x_{1}^{m}, y_{1}^{m}\right) \stackrel{=}{=} A\left(x_{1}^{m}, a_{1}^{(k-2) \cdot m}, y_{1}^{m}\right), \\
& \varphi\left(x_{1}^{m}\right) \stackrel{\text { def }}{=} A\left(\mathbf{e}\left(a_{1}^{(k-2) \cdot m}\right), x_{1}^{m}, a_{1}^{(k-2) \cdot m}\right) \text { and } \\
& c_{1}^{m} \stackrel{k}{=} A\left(\mathbf{e}\left(a_{1}^{(k-2) \cdot m}\right)\right)
\end{aligned}
$$

for all $x_{1}^{m}, y_{1}^{m} \in Q^{m}$, where $(Q ; A)$ is a $(k m, m)$-group, e its $\{1, n-m+1\}$-neutral operation and $k \geq 3$. [Cf. Th. 3.1-IV in [23].

c) If condition (c) from Th. 3.2 in $(Q ; A)$ holds, then $\varphi\left(x_{1}^{m}\right)=x_{1}^{m}$ for all $x_{1}^{m} \in Q^{m}$.

d) $(k m, m)$-groups $(k \geq 3, m \geq 2)$ with condition (0) from Th.6.1 exist, because $(2 m, m)$-groups exist and Th.6.2 holds. However, we do not know if $(k m, m)$-groups $(k \geq 3, m \geq 2)$ without condition (0) from Th.6.1 exist.

$9_{\bar{c}_{1}^{m}}^{m}=\left(c_{1}^{m}\right)^{-1}$ 
7. ON $(n, m)$-GROUPS FOR $n>2 m$ AND $n \neq k m$

7.1. Theorem [25]: Let $m \geq 2, s \geq 2,0<r<m, n=s \cdot m+r$ and let $(Q ; A)$ be an $(n, m)$-group. Also, let there exist a sequence $a_{1}^{k \cdot m-2 m}$, where $k=r-m+1$, such that for all $i \in\{0,1, \ldots, 2 m-1\}$, and for every $x_{1}^{2 m} \in Q$ the following equality holds

(0) $\stackrel{m}{A}\left(x_{1}^{i}, a_{1}^{k \cdot m-2 m}, x_{i+1}^{2 m}\right)=\stackrel{m}{A}\left(x_{1}^{2 m}, a_{1}^{k \cdot m-2 m}\right)$.

Then there are mapping $B$ of the set $Q^{2 m}$ into the set $Q^{m}, c_{1}^{m} \in Q^{m}$ and the sequence $\varepsilon_{1}^{(m-1)(n-m)}$ over $Q$ such that the following statements hold

(1) $(Q ; B)$ is a $(2 m, m)$-group;

(2) For all $j \in\{0, \ldots, m-1\}$ and for every $x_{1}^{m} \in Q$ the following equality holds

$$
B\left(x_{1}^{j}, c_{1}^{m}, x_{j+1}^{m}\right)=B\left(x_{1}^{m}, c_{1}^{m}\right) ;
$$

(3) For all $x_{1}^{m} \in Q$ the following equality holds

$$
A\left(x_{1}^{m}\right)=B\left(\stackrel{n-m}{B}\left(x_{1}^{n}, \varepsilon_{1}^{(m-1)(n-m)}\right), c_{1}^{m}\right) .
$$

(4) For all $t \in\{0, \ldots, m-1\}$ and for every $y_{1}^{r}, z_{1}^{m} \in Q$ the following equality holds

$$
{ }_{B}^{n-m-s+1}\left(y_{1}^{r}, z_{1}^{t}, \varepsilon_{1}^{(m-1)(n-m)}, z_{t+1}^{m}\right)=\stackrel{n-m-s+1}{B}\left(y_{1}^{r}, z_{1}^{m}, \varepsilon_{1}^{(m-1)(n-m)}\right) .
$$

Proof. Firstly we prove the following statements:

$1^{\circ}(Q, \stackrel{m}{A})$ is a $(k m, m)$-group, where $k=n-m+1$.

$2^{\circ} \quad$ Let $\mathrm{E}$ be a $\{1, k m-m+1\}$-neutral operation of $(k m, m)-\operatorname{group}(Q ; A)$. Also let
a) $B\left(x_{1}^{m}, y_{1}^{m}\right) \stackrel{\text { def }}{=} A\left(x_{1}^{m}, a_{1}^{k m-2 m}, y_{1}^{m}\right)$

for all $x_{1}^{m}, y_{1}^{m} \in Q^{m}$, where $a_{1}^{k m-2 m}$ from (0); and

Then:

$$
\text { b) } c_{1}^{m} \stackrel{\text { def }}{=} A\left(\overline{\mathrm{E}\left(a_{1}^{k m-2 m}\right)}\right) \text {. }
$$

1) $(Q ; B)$ is a $(2 m, m)$-group;

2) For all $x_{1}^{m} \in Q^{m}$ and for all $j \in\{0, \ldots, m-1\}$ the following equality holds

$$
B\left(x_{1}^{i}, c_{1}^{m}, x_{i+1}^{m}\right)=B\left(x_{1}^{m}, c_{1}^{m}\right) ; \text { and }
$$

3) For all $x_{1}^{k m} \in Q$ the following equality holds

$$
\stackrel{m}{A}\left(x_{1}^{k m}\right)=\stackrel{k}{B}\left(x_{1}^{k m}, c_{1}^{m}\right) .
$$

$3^{\circ} \quad$ Let e be a $\{1, n-m+1\}$-neutral operation of $(n, m)$-group $(Q ; A)$. Then for all $x_{1}^{m} \in Q$ and for every $\stackrel{(i)}{b}_{1}^{n-2 m}, i \in\{1, \ldots, m-1\}$, the following equality holds 


$$
A\left(x_{1}^{n}\right)=\stackrel{m}{A}\left(x_{1}^{n},\left.\quad \stackrel{(i)}{b_{1}^{n-2 m}, \mathbf{e}\left(\stackrel{(i)}{b}_{1}^{n-2 m}\right)}\right|_{i=1} ^{m-1}\right)
$$

$4^{\circ} \quad$ Let $\stackrel{(i)}{b_{1}^{n-2 m}}, i \in\{1, \ldots, m-1\}$, be an arbitrary sequence over $Q$. Also, let

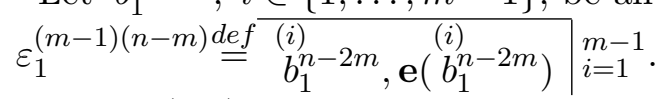

Then for all $x_{1}^{(s-1) m}, y_{1}^{r}, z_{1}^{m} \in Q$ and for all $j \in\{0, \ldots, m-1\}$ the following equality holds

$$
\stackrel{m}{A}\left(x_{1}^{(s-1) m}, y_{1}^{r}, z_{1}, \varepsilon_{1}^{(m-1)(n-m)}, z_{j+1}\right)=\stackrel{m}{A}\left(x_{1}^{(s-1) m}, y_{1}^{r}, z_{1}^{m}, \varepsilon_{1}^{(m-1)(n-m)}\right) .
$$

The proof of $1^{\circ}$ : By Prop. 4.7.

The proof of $2^{\circ}$ : By Th.6.1

Sketch of the proof of $3^{\circ}$ :

a) $m=2$ :

$\stackrel{2}{A}\left(x_{1}^{n}, b_{1}^{n-2 m}, \mathbf{e}\left(b_{1}^{n-2 m}\right)\right) \stackrel{4.3}{=}$

$A\left(A\left(x_{1}^{n}\right), b_{1}^{n-2 m}, \mathbf{e}\left(b_{1}^{n-2 m}\right)\right) \stackrel{2.1}{=} A\left(x_{1}^{n}\right)$

b) $m>2$ :

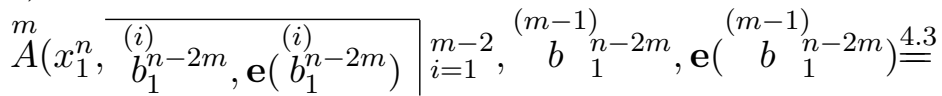

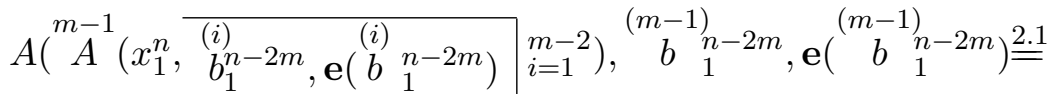

$\stackrel{m-1}{A}\left(x_{1}^{n}, \stackrel{(i)}{b_{1}^{n-2 m}}, \mathbf{e}\left(\stackrel{(i)}{b_{1}^{n-2 m}}\right){ }_{i=1}^{m-2}\right)=\ldots \stackrel{2.1}{=} A\left(x_{1}^{n}\right)$.

Sketch of the proof of $4^{\circ}$ [to the case $m=3, n=7$ ]:

$\stackrel{3}{A}\left(x_{1}^{3}, y, z_{1}^{3}, b, \mathbf{e}(b), c, \mathbf{e}(c)\right) \stackrel{4.4}{=}$

$\stackrel{2}{A}\left(x_{1}^{3}, y, A\left(z_{1}^{3}, b, \mathbf{e}(b)\right), c, \mathbf{e}(c)\right) \stackrel{2.1,2.11}{=}$

$\stackrel{2}{A}\left(x_{1}^{3}, y, A\left(z_{1}^{i}, b, \mathbf{e}(b), z_{i+1}^{3}\right), c, \mathbf{e}(c)\right)=$

$\left.\stackrel{2}{A}\left(x_{1}^{3}, y, A\left(z_{1}^{i}, b, \overline{\mathbf{e}_{j}(b)}\right]_{j=1}^{3}, z_{i+1}^{3}\right), c, \mathbf{e}(c)\right)=$

$\left.\stackrel{2}{A}\left(x_{1}^{3}, y, A\left(z_{1}^{i}, b,{\mathbf{\mathbf { e } _ { j } ( b )}}_{j=1}^{3-i}, \overline{\mathbf{e}_{j}(b)}\right]_{j=3-i+1}^{3}, z_{i+1}^{3}\right), c, \mathbf{e}(c)\right) \stackrel{4.4}{=}$

$\left.\left.\stackrel{2}{A}\left(x_{1}^{3}, y, z_{1}^{i}, b, \overline{\mathbf{e}_{j}(b)}\right]_{j=1}^{3-i}, A\left(\overline{\mathbf{e}_{j}(b)}\right]_{j=3-i+1}^{3}, z_{i+1}^{3}, c, \mathbf{e}(c)\right)\right) \stackrel{2.1,2.11}{=}$

$\left.\stackrel{2}{A}\left(x_{1}^{3}, y, z_{1}^{i}, b, \overline{\mathbf{e}_{j}(b)}\right]_{j=1}^{3-i}, A\left(\overline{\mathbf{e}_{j}(b)}{ }_{j=3-i+1}^{3}, c, \mathbf{e}(c), z_{i+1}^{3}\right)\right) \stackrel{4.4}{=}$

$\left.\stackrel{3}{A}\left(x_{1}^{3}, y, z_{1}^{i}, b, \overline{\mathbf{e}_{j}(b)}\right]_{j=1}^{3-i},\left.\overline{\mathbf{e}_{j}(b)}\right|_{j=3-i+1} ^{3}, c, \mathbf{e}(c), z_{i+1}^{3}\right)=$

$\stackrel{3}{A}\left(x_{1}^{3}, y, z_{1}^{i}, b, \mathbf{e}(b), c, \mathbf{e}(c), z_{i+1}^{3}\right)$.

By $1^{\circ}$ and $2^{\circ}$, we have (1) and (2). 
Sketch of the proof of (3): By $\left.2^{\circ}[: 3)\right]$ and by $3^{\circ}$.

$\left(k=n-m+1,\left.\varepsilon_{1}^{(m-1)(n-m) \stackrel{\text { def }}{=} \stackrel{(i)}{b}{ }_{1}^{n-2 m}, \mathbf{e}\left(\stackrel{(i)}{b}{ }_{1}^{n-2 m}\right)}\right|_{i=1} ^{m-1}.\right)$

Sketch of the proof of (4):

$\stackrel{m}{A}\left(x_{1}^{(s-1) m}, y_{1}^{r}, z_{1}^{m}, \varepsilon_{1}^{(m-1)(n-m)}\right) \stackrel{4^{\circ}}{=}$

$\stackrel{m}{A}\left(x_{1}^{(s-1) m}, y_{1}^{r}, z_{1}^{j}, \varepsilon_{1}^{(m-1)(n-m)}, z_{j+1}^{m}\right) \stackrel{\left.4^{\circ}-3\right)}{\Longrightarrow}$

$\stackrel{k}{B}\left(x_{1}^{(s-1) m}, y_{1}^{r}, z_{1}^{m}, \varepsilon_{1}^{(m-1)(n-m)}, c_{1}^{m}\right)=$

$\stackrel{k}{B}\left(x_{1}^{(s-1) m}, y_{1}^{r}, z_{1}^{j}, \varepsilon_{1}^{(m-1)(n-m)}, z_{j+1}^{m}, c_{1}^{m}\right) \stackrel{1^{\circ}, 4.5}{\Longrightarrow}$

$\stackrel{s}{B}\left(x_{1}^{(s-1) m}, \stackrel{n-m-s+1}{B}\left(y_{1}^{r}, z_{1}^{m}, \varepsilon_{1}^{(m-1)(n-m)}\right), c_{1}^{m}\right)=$

$\stackrel{s}{B}\left(x_{1}^{(s-1) m}, \stackrel{n-m-s+1}{B}\left(y_{1}^{r}, z_{1}^{j}, \varepsilon_{1}^{(m-1)(n-m)}, z_{j+1}^{m}\right), c_{1}^{m}\right) \stackrel{1^{\circ}, 4.7}{\Longrightarrow}$

${ }_{B}^{n-m-s+1}\left(y_{1}^{r}, z_{1}^{m}, \varepsilon_{1}^{(m-1)(n-m)}\right)={ }^{n-m-s+1}\left(y_{1}^{r}, z_{1}^{j}, \varepsilon_{1}^{(m-1)(n-m)}, z_{j+1}^{m}\right)$.

The proof of Th. 7.1 is completed.

7.2. Theorem [25]: Let $(Q ; B)$ be a $(2 m, m)$-group and $m \geq 2$. Also let:

(a) $c_{1}^{m}$ be an element of the set $Q^{m}$ such that for every $i \in\{0, \ldots, m-1\}$, and for every $x_{1}^{m} \in Q$ the following equality holds

$B\left(x_{1}^{i}, c_{1}^{m}, x_{i+1}^{m}\right)=B\left(x_{1}^{m}, c_{1}^{m}\right) ;$ and

(b) $\varepsilon_{1}^{(m-1)(n-m)}$ be a sequence over $Q$ such that for all $j \in\{0, \ldots, m-1\}$, and for every $y_{1}^{r}, z_{1}^{m} \in Q$ the following equality holds

$\stackrel{n-m-s+1}{B}\left(y_{1}^{r}, z_{1}^{j}, \varepsilon_{1}^{(m-1)(n-m)}, z_{j+1}^{m}\right)=\stackrel{n-m-s+1}{B}\left(y_{1}^{r}, z_{1}^{m}, \varepsilon_{1}^{(m-1)(n-m)}\right)$,

where $s \geq 2,0<r<m$ and $n=s \cdot m+r$.

Further on, let

(c) $A\left(x_{1}^{m}\right) \stackrel{\text { def }}{=} B\left(\stackrel{n-m}{B}\left(x_{1}^{n}, \varepsilon_{1}^{(m-1)(n-m)}\right), c_{1}^{m}\right)$

for all $x_{1}^{n} \in Q$.

Then $(Q ; A)$ is an $(n, m)$-group.

Proof. Firstly we prove the following statements:

1 The $<1,2>$-associative law holds in $(Q ; A)$.

$\stackrel{\circ}{2}$ For every $a_{1}^{n} \in Q$ there is exactly one $x_{1}^{m} \in Q^{m}$ such that the following equality holds

$A\left(x_{1}^{m}, a_{1}^{n-m}\right)=a_{n-m+1}^{n}$.

$\stackrel{\circ}{3}(Q ; A)$ is an $(n, m)$-group.

4 For every $a_{1}^{n} \in Q$ there is exactly one $y_{1}^{m} \in Q^{m}$ such that the following equality holds 
$A\left(a_{1}^{n-m}, y_{1}^{m}\right)=a_{n-m+1}^{n}$.

Sketch of the proof of 1 :

a) $A\left(A\left(x_{1}^{n}\right), x_{n+1}^{2 n-m}\right) \stackrel{(c)}{=}$

$\stackrel{n-m+1}{B}\left(\stackrel{n-m+1}{B}\left(x_{1}^{n}, \varepsilon_{1}^{(m-1)(n-m)}, c_{1}^{m}\right), x_{n+1}, x_{n+2}^{2 n-m}, \varepsilon_{1}^{(m-1)(n-m)}, c_{1}^{m}\right) \stackrel{4.5}{=}$

$\stackrel{n-m+1}{B}\left(x_{1}, \stackrel{n-m}{B}{ }^{n+1}\left(x_{2}^{n}, \varepsilon_{1}^{(m-1)(n-m)}, c_{1}^{m}, x_{n+1}\right), x_{n+2}^{2 n-m}, \varepsilon_{1}^{(m-1)(n-m)}, c_{1}^{m}\right)$.

b) $\stackrel{n-m+1}{B}\left(x_{2}^{n}, \varepsilon_{1}^{(m-1)(n-m)}, c_{1}^{m}, x_{n+1}\right) \stackrel{4.4}{=}$

$\stackrel{n-m}{B}\left(x_{2}^{n}, \varepsilon_{1}^{(m-1)(n-m-1)}, B\left(\varepsilon_{(m-1)(n-m+1)+1}^{(m-1)(n-m)}, c_{1}^{m}, x_{n+1}\right)\right) \stackrel{(a)}{=}$

$\stackrel{n-m}{B}\left(x_{2}^{n}, \varepsilon_{1}^{(m-1)(n-m-1)}, B\left(\varepsilon_{(m-1)(n-m+1)+1}^{(m-1)(n-m)}, x_{n+1}, c_{1}^{m}\right)\right) \stackrel{4.4}{=}$

$\stackrel{n-m+1}{B}\left(x_{2}^{n}, \varepsilon_{1}^{(m-1)(n-m-1)}, \varepsilon_{(m-1)(n-m+1)+1}^{(m-1)(n-m)}, x_{n+1}, c_{1}^{m}\right)=$

$\stackrel{n-m+1}{B}\left(x_{2}^{n}, \varepsilon_{1}^{(m-1)(n-m-1)}, x_{n+1}, c_{1}^{m}\right) \stackrel{4.5}{=}$

$\stackrel{s}{B}\left(x_{2}^{(s-1) m+1}, \stackrel{n-m-s+1}{B}\left(x_{(s-1) m+2}^{n},{ }^{10} \varepsilon_{1}^{(m-1)(n-m)}, x_{n+1}\right), c_{1}^{m}\right) \stackrel{(b)}{=}$

$\stackrel{s}{B}\left(x_{2}^{(s-1) m+1}, \stackrel{n-m-s+1}{B}\left(x_{(s-1) m+2}^{n}, x_{n+1}, \varepsilon_{1}^{(m-1)(n-m)}\right), c_{1}^{m}\right)=$

$\stackrel{s}{B}\left(x_{2}^{(s-1) m+1}, \stackrel{n-m-s+1}{B}\left(x_{(s-1) m+2}^{n+1}, \varepsilon_{1}^{(m-1)(n-m)}\right), c_{1}^{m}\right) \stackrel{4.5}{=}$

$\stackrel{n-m+1}{B}\left(x_{2}^{(s-1) m+2}, x_{(s-1) m+2}^{n+1}, \varepsilon_{1}^{(m-1)(n-m)}, c_{1}^{m}\right)=$

$\stackrel{n-m+1}{B}\left(x_{2}^{n+1}, \varepsilon_{1}^{(m-1)(n-m)}, c_{1}^{m}\right) \stackrel{(c)}{=} A\left(x_{2}^{n+1}\right)$.

Finally, by $a), b$ ) and by $(c)$, we obtain 1 .

Sketch of the proof of $\stackrel{\circ}{2}$ :

$A\left(x_{1}^{m}, a_{1}^{n-m}\right)=a_{n-m+1}^{n} \stackrel{(c)}{\Longleftrightarrow}$

$\stackrel{n-m+1}{B}\left(x_{1}^{m}, a_{1}^{n-m}, \varepsilon_{1}^{(m-1)(n-m)}, c_{1}^{m}\right)=a_{n-m+1}^{n} \stackrel{4.5}{\Longleftrightarrow}$

$B\left(x_{1}^{m}, \stackrel{n-m}{B}\left(a_{1}^{n-m}, \varepsilon_{1}^{(m-1)(n-m)}, c_{1}^{m}\right)\right)=a_{n-m+1}^{n}$.

The proof of $\stackrel{\circ}{3}$ : By $\stackrel{\circ}{1}, \stackrel{\circ}{2}$ and Prop. $4.2_{1}$.

Sketch of the proof of $\stackrel{\circ}{4}$ :

$A\left(a_{1}^{n-m}, x_{1}^{m}\right)=a_{n-m+1}^{n} \stackrel{(c)}{\Longleftrightarrow}$
$\stackrel{n-m+1}{B}\left(a_{1}^{n-m}, y_{1}^{m}, \varepsilon_{1}^{(m-1)(n-m)}, c_{1}^{m}\right)=a_{n-m+1}^{n}$.

Whence, by Prop. 4.7 and by Def. 1.1, we obtain $\stackrel{\circ}{4}$.

Finally, by $\stackrel{\circ}{2}-\stackrel{\circ}{4}$ and by Prop. 4.1 , we conclude that Th. 7.2 holds.

$10_{n}=s m+r$. 


\section{SKEW OPERATION ON $(n, m)$-GROUPS}

8.1. Definition [28]: Let $(Q ; A)$ be an $(n, m)$-group and $n \geq 2 m+1$. Further on, let - be a mapping of the set $Q$ into the set $Q^{m}$. Then, we shall say that mapping ${ }^{-}$is a skew operation of the $(n, m)$-group $(Q ; A)$ iff for each $a \in Q$ there is (exactly one) $\bar{a} \in Q^{m}$ such that the following equality holds

$$
A(\stackrel{n-m}{a}, \bar{a})=\stackrel{m}{a} 11 .
$$

Remark: For $m=1$ skew operation is introduced in [6].

8.2. Proposition: Let $(Q ; A)$ be an $(n, m)$-group and $n \geq 2 m+1$. Then for all $i \in\{1, \ldots, n-m+1\}$ and for every $a \in Q$ the following equality holds

$$
A(\stackrel{i-1}{a}, \bar{a}, \stackrel{n-(i-1+m)}{a})=\stackrel{m}{a} .
$$

\section{Sketch of the proof.}

$A(\stackrel{n-m}{a}, \bar{a}) \stackrel{(0)}{=} \stackrel{m}{a} \Rightarrow$

$A(\stackrel{i-1}{a}, A(\stackrel{n-m}{a}, \bar{a}), \stackrel{n-(i-1+m)}{a})=A(\stackrel{i-1}{a}, \stackrel{m}{a}, \stackrel{n-(i-1+m)}{a}) \Rightarrow$

$A\left({ }^{i-1} a, A\left({ }^{n-m} a, \bar{a}\right), \stackrel{n-(i-1+m)}{a}\right)=A(\stackrel{n}{a}) \stackrel{1.1(\mid)}{\Longrightarrow}$

$A(\stackrel{i-1}{a}, \underset{a-(i-1+m)}{a}, A(\stackrel{i-1}{a}, \bar{a}, \stackrel{n-(i-1+m)}{a}))=A(\stackrel{n}{a}) \Rightarrow$

$A(\stackrel{n-m}{a}, A(\stackrel{i-1}{a}, \bar{a}, \stackrel{n-(i-1+m)}{a}))=A(\stackrel{n-m}{a}, \stackrel{m}{a}) \stackrel{1.1(\|)}{\Longrightarrow}$

$A(\stackrel{i-1}{a}, \bar{a}, \stackrel{n-(i-1+m)}{a})=\stackrel{m}{a}$.

8.3. Proposition [28]: Let $(Q ; A)$ be an $(n, m)$-group and $n \geq 2 m+1$. Then for all $a, x_{1}^{m} \in Q$ the equality

$$
A\left(x_{1}^{m},{ }^{n-2 m}, \bar{a}\right)=x_{1}^{m}
$$

holds.

\section{Sketch of the proof.}

$$
\begin{aligned}
& A\left(x_{1}^{m}, \stackrel{n-2 m}{a}, \bar{a}\right)=y_{1}^{m} \Rightarrow \\
& A\left(A\left(x_{1}^{m}, \stackrel{n-2 m}{a}, \bar{a}\right), \stackrel{n-m}{a}\right)=A\left(y_{1}^{m}, \stackrel{n-m}{a}\right) \stackrel{1.1(\mid)}{\Longrightarrow} \\
& A\left(x_{1}^{m}, \stackrel{n-2 m}{a}, A\left(\bar{a},{ }^{n-m} a\right)\right)=A\left(y_{1}^{m}, \stackrel{n-m}{a}\right) \stackrel{8.2, i=1}{\Longrightarrow} \\
& A\left(x_{1}^{m}, \stackrel{n-2 m}{a}, \stackrel{m}{a}\right)=A\left(y_{1}^{m}, \stackrel{n-m}{a}\right) \Rightarrow \\
& A\left(x_{1}^{m},{ }^{n-m} a\right)=A\left(y_{1}^{m}, \stackrel{n-m}{a}\right) \stackrel{1.1(\|)}{\Longrightarrow} x_{1}^{m}=y_{1}^{m} \text {. }
\end{aligned}
$$

8.4. Theorem [28]: Let $n \geq 2 m+1,(Q ; A)$ be an $(n, m)-$ group, e its $\{1, n-m+$ $1\}$-neutral operation and ${ }^{-}$its skew operation. Then for all $a \in Q$ the following equality holds

$$
\bar{a}=\mathbf{e}\left(\begin{array}{c}
n-2 m \\
a
\end{array}\right)
$$

\footnotetext{
${ }^{11}$ See Def. 1.1.-(||).
} 


\section{Sketch of the proof.}

$A\left(x_{1}^{m}, \stackrel{n-2 m}{a}, \bar{a}\right) \stackrel{8.3}{=} x_{1}^{m} \wedge A\left(x_{1}^{m}, \stackrel{n-2 m}{a}, \mathbf{e}(\stackrel{n-2 m}{a})\right) \stackrel{2.5}{=} x_{1}^{m} \Rightarrow$ $A\left(x_{1}^{m}, \stackrel{n-2 m}{a}, \bar{a}\right)=A\left(x_{1}^{m}, \stackrel{n-2 m}{a}, \mathbf{e}(\stackrel{n-2 m}{a})\right) \stackrel{1.1(\|)}{\Longrightarrow} \bar{a}=\mathbf{e}(\stackrel{n-2 m}{a})$.

8.5. Theorem [28]: Let $(Q ; A)$ be an $(n, m)$-group, e its $\{1, n-m+1\}$-neutral operation, - its skew operation and $n>3 m$. Then for every sequence $a_{1}^{n-m+1}$ over $Q$ the following equality holds

$$
\mathrm{E}\left(\stackrel{m}{a}_{1}, \ldots, \stackrel{m}{a_{n-m+1}}\right)=\stackrel{n-2 m-1}{A}\left(\bar{a}_{n-m-1}, \stackrel{n-3 m}{a_{n-m+1}}, \ldots, \bar{a}_{1},{ }^{n-3 m} a_{1}\right)^{12},
$$

where $\mathrm{E}$ is the $\{1, m(n-m)+1\}$-neutral operation of $(m(n-m)+m, m)$-group $(Q ; \stackrel{m}{A})$.

\section{Sketch of the proof.}

$\left.{ }^{m} \stackrel{n-2 m-1}{A}\left(\bar{a}_{n-m-1}, \stackrel{n-3 m}{a_{n-m+1}}, \ldots, \bar{a}_{1}, \stackrel{n-3 m}{a_{1}}\right), \stackrel{m}{a}_{1}, \ldots, \stackrel{m}{a_{n-m-1}}, x_{1}^{m}\right) \stackrel{8.4}{=}$

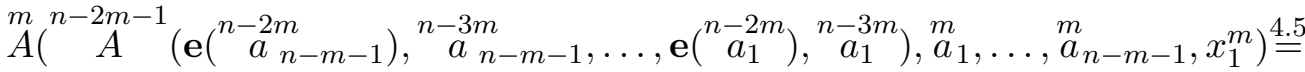

$\stackrel{n-m-1}{A}\left(\mathbf{e}\left(\stackrel{n-2 m}{a}_{n-m-1}\right), \stackrel{n-3 m}{a_{n-m-1}}, \ldots, \mathbf{e}\left({ }^{n-2 m} a_{1}\right),{ }^{n-3 m} a_{1}, m_{1}, \ldots, \stackrel{m}{a}_{n-m-1}, x_{1}^{m}\right) \stackrel{4.4}{=}$

$\stackrel{n-m-2}{A}\left(\mathbf{e}\left(\stackrel{n-2 m}{a}_{n-m-1}\right),{ }^{n-3 m} a_{n-m-1}, \ldots, A\left(\mathbf{e}\left({ }^{n-2 m} a_{1}\right),{ }^{n-3 m} a_{1}, \stackrel{m}{a}_{1}, \stackrel{m}{a}_{2}\right), \stackrel{m}{a}_{3}, \ldots, \stackrel{m}{a}_{n-m-1}, x_{1}^{m}\right) \stackrel{2.1}{=}$ $\stackrel{n-m-2}{A}\left(\mathbf{e}\left(\stackrel{n-2 m}{a_{n-m-1}}\right), \stackrel{n-3 m}{a}{ }_{n-m-1}, \ldots, \stackrel{m}{a}_{2}, \stackrel{m}{a}_{3}, \ldots, \stackrel{m}{a}_{n-m-1}, x_{1}^{m}\right)=$

$A\left(\mathbf{e}\left(\stackrel{n-2 m}{a}_{n-m-1}\right), \stackrel{n-3 m}{a}{ }_{n-m-1}, \stackrel{m}{a}_{n-m-1}, x_{1}^{m}\right)=$

$A\left(\mathbf{e}\left(\stackrel{n-2 m}{a_{n-m-1}}\right), \stackrel{n-2 m}{a}{ }_{n-m-1}, x_{1}^{m}\right) \stackrel{2.1}{=} x_{1}^{m}$.

Hence, by Prop. 4.7 and Th.2.5, we conclude that for every sequence $a_{1}^{n-m-1}$ over $Q$ and for all $x_{1}^{m} \in Q^{m}$ the following equality holds

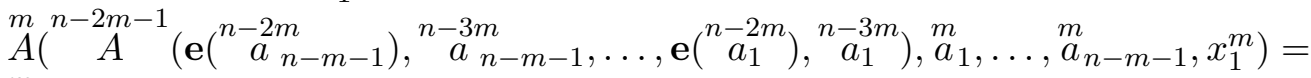
$\stackrel{m}{A}\left(\mathrm{E}\left(\stackrel{m}{a}_{1}, \ldots, \stackrel{m}{a_{n-m-1}}\right), \stackrel{m}{a_{1}}, \ldots, \stackrel{m}{a_{n-m-1}}, x_{1}^{m}\right)$,

where $\mathrm{E}$ is the $\{1, m(n-m)+1\}$-neutral operation of $(m(n-m)+m, m)$-group $(Q ; \stackrel{m}{A})$.

Finaly, whence, by Def. 1.1, we conclude that the proposition holds.

For $m=1$ Th.8.5 is reduced to:

8.6. Theorem [30]: Let $(Q ; A)$ be an $n$-group, e its $\{1, n\}$-neutral operation, its skew operation and $n>3$. Then for every sequence $a_{1}^{n-2}$ over $Q$ the following equality holds

$$
\begin{aligned}
& \mathbf{e}\left(a_{1}^{n-2}\right)={ }^{n-3}\left(\bar{a}_{n-2}, \quad{ }^{n-3} a_{n-2}, \ldots, \bar{a}_{1},{ }^{n-3} a_{1}\right) . \\
& 12 n>3 m \Rightarrow{ }_{i}^{n-3 m} \neq \emptyset(i \in\{1, \ldots, n-m-1\}) .
\end{aligned}
$$


Remark: See, also VIII-2.9 and Appendix 2 in [23].

8.7. Remark: In [Ušan 1998] topological $n$-groups for $n \geq 2$ are defined on $n$-groups as algebras $\left(Q ; A,{ }^{-1}\right)$ of the type $<n, n-1>$ [15], [17]; cf. Ch. III and Ch. IX in [23] ]. In [29] topological $n$-groups for $n \geq 3$ are considered on $n$-groups as algebras $\left(Q ; A,{ }^{-}\right)$of the type $<n, 1>$ [ [10]]. In [Ušan 1998] it is proved that for $n \geq 3$ these definitions are mutually equivalent. The key roole in the proof had Theorem 8.6. About topological n-groups see, also, Chapter VIII in [23]. Topological $(n, m)$-groups are not defined.

\section{REFERENCES}

[1] Ǵ. Čupona, Vector valued semigroups, Semigroup Forum, 26 (1983), 65-74.

[2] Ǵ. Čupona and D. Dimovski, On a class of vector valued groups, Proceedings of the Conf. "Algebra and Logic", Zagreb 1984, 29-37.

[3] Ǵ. Cupona, N. Celakoski, S. Markovski and D. Dimovski, Vector valued groupoids, semigroups and groups, in: Vector valued semigroups and groups, (B. Popov, Ǵ. Čupona and N. Celakoski, eds.), Skopje 1988, 1-78.

[4] D. Dimovski and S. Ilić, Commutative $(2 m, m)$-groups, in: Vector valued semigroups and groups, (B. Popov, Ǵ. Čupona and N. Celakoski, eds.), Skopje 1988, 79-90.

[5] D. Dimovski and K. Trenčevski, One-dimensional (4,2)-Lie groups, in: Vector valued semigroups and groups, (B. Popov, G. Čupona and N. Celakoski, eds.), Skopje 1988, 91-102.

[6] ] W. Dörnte, Untersuchengen über einen verallgemeinerten Gruppenbegriff, Math. Z., 29 (1928), 1-19.

[7] W. A. Dudek, Varieties of polyadic groups, Filomat (Niš), 9 (1995), No. 3, 657-674.

[8] R. Galić, On (n,m)-groups for $n>2 m$, Sarajevo J. Math., Vol. 1(14) (2005), 171-174.

[9] R. Galić and A. Katić, On neutral operations of ( $n, m)$-groups, Math. Moravica, 9 (2005), $1-3$.

[10] B. Gleichgewicht, K. Glazek, Remarks on n-groups as abstract algebras, Colloq. Math., 17(1967), 209-219.

[11] L. M. Gluskin, Position operatives, (Russian), Mat. Sb., t., 68(110), No. 3 (1965), 444-472.

[12] M. Hosszú, On the explicit form of n-group operations, Publ. Math. (Debrecen), 10 (1963), $88-92$.

[13] J. Ušan, Neutral operations of n-groupoids, (Russian), Rev. of Research, Fac. of Sci. Univ. of Novi Sad, Math. Ser., 18, No. 2 (1988), 117-126.

[14] J. Ušan, Neutral operations of $(n, m)$-groupoids, (Russian), Rev. of Research, Fac. of Sci. Univ. of Novi Sad, Math. Ser., 19, No. 2 (1989), 125-137.

[15] J. Ušan, A comment on n-groups,Rev. of Research, Fac. of Sci. Univ. of Novi Sad, Math. Ser., 24, No. 1 (1994), 281-288. 
[16] J. Ušan, On Hosszú-Gluskin Algebras corresponding to the same n-group, Rev. of Research, Fac. of Sci. Univ. of Novi Sad, Math. Ser., 25, No. 1 (1995), 101-119.

[17] J. Ušan, $n$-groups as variety of type $\langle n, n-1, n-2\rangle$, in: Algebra and Model Theory, (A.G. Pinus and K.N. Ponomaryov, eds.), Novosibirsk 1997, 182-208.

[18] J. Ušan, On n-groups, Maced. Acad. Sci. and Arts, Contributions, Sect. Math. Techn. Sci., XVIII 1-2 (1997), 17-20.

[19] J. Ušan, Note on (n,m)-groups, Math. Moravica, 3 (1999), 127-139.

[20] J. Ušan, A note on $n$-groups for $n \geq 3$, Novi Sad J. Math., 29, No. 1 (1999), 55-59.

[21] J. Ušan, On (n,m)-groups, Math. Moravica, 4 (2000), 115-118.

[22] J. Ušan, A comment on ( $n, m)$-groups for $n \geq 3 m$, Math. Moravica, 5 (2001), 159-162.

[23] J. Ušan, n-groups in the light of the neutral operations, Math. Moravica, Special Vol. (2003), monograph (Electronic version - 2006: http://www.moravica.tfc.kg.ac.yu).

[24] J. Ušan, About a class of (n,m)-groups, Math. Moravica, Vol. 9 (2005), 77-86.

[25] J. Ušan, A comment on (n,m)-groups, Novi Sad J. Math., 35, No. 2 (2005), 133-141.

[26] J. Ušan and A. Katić, Two characterizations of $(n, m)$-groups for $n \geq 3 m$, Math. Moravica, 8-1 (2004), 73-78.

[27] J. Ušan and M. Žižović, On (n,m)-groups for $n \geq 3 m$, Math. Moravica, Vol. 9 (2005), $87-93$.

[28] J. Ušan and M. Žižović, Skew operation on $(n, m)$-groups, submitted.

[29] M. Žižović, Topological analogy of Hosszú-Gluskin Theorem, (Serbo-Croatian), Mat. Vesnik, 13(28) (1976), 233-235.

[30] M. Žižović, On $\{1, n\}$-neutral, inversing and skew operations of $n$-groups, Math. Moravica, 2 (1998), 169-173.

Department of Mathematics

AND INFORMATICS

UNIVERSITY OF NOVI SAD

Trg D. Obradovića 4

21000 NOVI SAD

SERBIA AND MONTENEGRO 